\title{
Pellino3 ubiquitinates RIP2 and mediates Nod2- induced signaling and protective effects in colitis
}

\author{
Shuo Yang ${ }^{1}$, Bingwei Wang ${ }^{1}$, Fiachra Humphries ${ }^{1}$, Ruaidhri Jackson ${ }^{1}$, Marc E Healy ${ }^{1}$, Ronan Bergin ${ }^{1}$, \\ Gabriella Aviello ${ }^{2}$, Barry Hall ${ }^{3}$, Deirdre McNamara ${ }^{3}$, Trevor Darby ${ }^{4}$, Aoife Quinlan ${ }^{4}$, Fergus Shanahan ${ }^{4}$, \\ Silvia Melgar ${ }^{4}$, Padraic G Fallon ${ }^{2,5,6} \&$ Paul N Moynagh ${ }^{1}$
}

\begin{abstract}
Mutations that result in loss of function of Nod2, an intracellular receptor for bacterial peptidoglycan, are associated with Crohn's disease. Here we found that the E3 ubiquitin ligase Pellino3 was an important mediator in the Nod2 signaling pathway. Pellino3deficient mice had less induction of cytokines after engagement of Nod2 and had exacerbated disease in various experimental models of colitis. Furthermore, expression of Pellino3 was lower in the colons of patients with Crohn's disease. Pellino3 directly bound to the kinase RIP2 and catalyzed its ubiquitination. Loss of Pellino3 led to attenuation of Nod2-induced ubiquitination of RIP2 and less activation of the transcription factor NF-кB and mitogen-activated protein kinases (MAPKs). Our findings identify RIP2 as a substrate for Pellino3 and Pellino3 as an important mediator in the Nod2 pathway and regulator of intestinal inflammation.
\end{abstract}

The innate immune system is equipped with pattern-recognition receptors that recognize pathogen-associated molecular patterns ${ }^{1}$. Pattern-recognition receptors include transmembrane Toll-like receptors (TLRs) and cytosolic Nod-like receptors ${ }^{2}$. Nod1 and Nod2 recognize structures in bacterial peptidoglycan ${ }^{3}$. Loss-of-function mutants of Nod2 are associated with Crohn's disease ${ }^{4-6}$, whereas gain-offunction mutants result in predisposition to the development of earlyonset sarcoidosis and Blau syndrome $e^{7,8}$. Nod2 responds to muramyl dipeptide (MDP), a derivative of peptidoglycan ${ }^{9,10}$. Nod2 consists of two amino-terminal caspase-recruitment domains (CARDs), a central self-oligomerization NACHT region and multiple carboxyterminal leucine-rich repeats ${ }^{11}$. Engagement of the leucine-rich repeats by MDP promotes a conformational change that exposes the NACHT domain, which allows self-oligomerization of Nod2 and the binding of its CARDs to the CARD-containing kinase RIP2 (refs. 12,13). RIP2 interacts with the kinase TAK1, which leads to activation of the transcription factor NF- $\mathrm{KB}$ and mitogen-activated protein kinases (MAPKs) and induction of the expression of proinflammatory cytokines ${ }^{14-18}$. Ubiquitination of RIP2 is critical for Nod2 signaling pathways ${ }^{15,16}$.

The attachment of polyubiquitin chains to RIP2 serves to recruit TAK1 via the adaptors TAB2 and TAB3 (ref. 19), and that facilitates TAK1-induced phosphorylation and activation of IKB kinases (IKKs) that induce phosphorylation of inhibitory I $\mathrm{KB}$ proteins ${ }^{20}$. Phosphorylated $\mathrm{I} \kappa \mathrm{B}$ proteins undergo proteasome-mediated degradation $^{21}$ that allows NF- $\kappa \mathrm{B}$ to translocate to the nucleus and induce proinflammatory gene expression ${ }^{22}$. Studies have investigated the enzymes responsible for catalyzing the ubiquitination of
RIP2. The Ubc13-Uev1a dimer acts as the E2 conjugating enzyme in the Lys63 (K63)-linked polyubiquitination of RIP2 (refs. 15,16), but the identity of the E3 ubiquitin ligase(s) that directly ubiquitinate(s) RIP2 to mediate Nod2-induced activation of NF- $\kappa B$ remains unclear. TRAF6 has been proposed as the main E3 ligase for RIP2 (ref. 15), but the ubiquitination of RIP2 is intact in TRAF6-deficient cells ${ }^{16}$ and knockdown of TRAF6 does not affect RIP2-mediated activation of NF$\kappa B^{9,14}$. Three members of the 'IAP' family of E3 ubiquitin ligases (XIAP, cIAP1 and CIAP2) have been proposed to regulate RIP2 ubiquitination $^{23,24}$. Although the conclusions of the last two studies differ about the functional importance of cIAP1 and cIAP2 in mediating Nod2induced ubiquitination of RIP2, one demonstrated that XIAP promotes the ubiquitination of RIP2 and recruitment of the linear ubiquitin chain-assembly complex (LUBAC) to Nod2 (ref. 23). However, the XIAP-mediated polyubiquitination of RIP2 is not K63 linked, a type of linkage associated with RIP2-induced activation of NF- $\mathrm{KB}$. The E3 ligase Itch can also directly ubiquitinate RIP2 to negatively regulate Nod2-induced activation of NF- $\mathrm{KB}^{25}$. Thus, it remains unclear which E3 ubiquitin ligase directly catalyzes the K63-linked ubiquitination of RIP2 and mediates the Nod2-induced activation of NF- $\kappa B$.

Pellino1, Pellino2 and two spliced forms of Pellino3 (Pellino3 long (Pellino31; also called Pellino3a) and Pellino3 short (Pellino3s; also called Pellino3b)) constitute a family of E3 ubiquitin ligases ${ }^{26,27}$. They contain a phosphorylated threonine-binding amino-terminal forkhead-associated (FHA) domain and a carboxy-terminal RING-like domain that facilitate the recruitment and K63-linked polyubiquitination, respectively, of kinases of the IRAK family, which are signaling intermediates in TLR pathways ${ }^{28-31}$. Pellino proteins undergo

1Institute of Immunology, Department of Biology, National University of Ireland Maynooth, Maynooth, Ireland. ${ }^{2}$ Trinity Biomedical Sciences Institute, Trinity College Dublin, Dublin, Ireland. ${ }^{3}$ Department of Gastroenterology, Adelaide and Meath Hospital \& Clinical Medicine, Trinity College Dublin, Dublin, Ireland. ${ }^{4}$ Alimentary Pharmabiotic Centre, University College Cork, Cork, Ireland. ${ }^{5}$ Institute of Molecular Medicine, School of Medicine, Trinity College Dublin, Dublin, Ireland. ${ }^{6}$ National Children's Research Centre, Our Lady's Children's Hospital, Dublin, Ireland. Correspondence should be addressed to P.N.M. (paul.moynagh@nuim.ie). 
phosphorylation that regulates their E3 ligase activity ${ }^{31-35}$. Pellino1 is a key mediator of NF- $\kappa \mathrm{B}$ activation in the TLR3 and TLR4 signaling pathways ${ }^{36}$ and is a negative regulator of $\mathrm{T}$ cell activation ${ }^{37,38}$. Pellino3 negatively regulates the TLR3-induced expression of type I interferons ${ }^{39}$. We identify Pellino3 here as an important mediator in the Nod2 pathway. We found lower expression of Pellino3 protein in the colons of patients with Crohn's disease and diminished signaling pathways and colitis-protective effects of Nod2 in Pellino3-deficient mice. We propose that Pellino3 acts as a direct E3 ubiquitin ligase for RIP2 and thus facilitates the Nod2-mediated activation of NF- $\kappa B$ and downstream gene expression.

\section{RESULTS}

\section{Pellino3 deficiency diminishes Nod2 signaling}

We studied Pellino3-deficient $\left(\right.$ Peli3 $^{-/-}$) mice $^{39}$ to assess the role of Pellino3 in activation of the Nod2 pathway. We obtained bone marrow-derived macrophages (BMDMs) from wild-type, Peli3 ${ }^{-/-}$ and Nod2-deficient (Nod2 $2^{-I-}$ ) mice and compared their responsiveness to the Nod2 ligand MDP. Treatment of wild-type BMDMs with MDP resulted in higher expression of interleukin (IL-6), IL- $1 \beta$, tumor-necrosis factor (TNF), the p40 subunit of IL-12

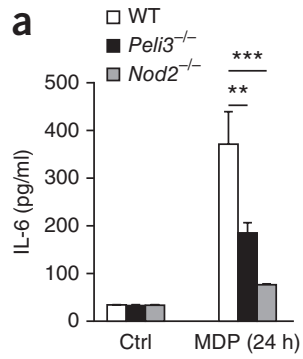

(IL-12p40) and the chemokine CXCL1, but such induction was absent in Nod2 ${ }^{-/-}$BMDMs and much lower in Peli3 ${ }^{-/}$BMDMs (Fig. 1a). MDP-induced expression of the mRNA encoding those cytokines was also absent from Nod2 $2^{-1-}$ BMDMs and was greatly diminished in Peli3 ${ }^{-/-}$BMDMs (Fig. 1b). We next assessed the effect of Pellino3 deficiency on the ability of Nod2 to act together with TLRs. Using expression of mRNA encoding IL-12p40 as a 'readout', we found that MDP enhanced the effects of the TLR4 ligand lipopolysaccharide (LPS) and the TLR2 ligand $\mathrm{Pam}_{3} \mathrm{CSK}_{4}$ in wild-type BMDMs but not in Peli3 ${ }^{-1-}$ BMDMs (Fig. 1c). Nod2 promoted the time-dependent activation of NF- $\mathrm{KB}$ and the three MAPK pathways (Jnk, p38 and Erk) in wild-type BMDMs, as indicated by the MDP-induced phosphorylation of $\mathrm{I} \kappa \mathrm{B} \alpha$ and of Jnk, p38 and Erk, respectively, whereas the activation of all four pathways was lower in Peli3-/- BMDMs (Fig. 1d). We next addressed the role of Pellino3 as a mediator of the Nod2 pathway in human cells. An NF- $\kappa B$-regulated luciferase reporter gene was induced by expression of Nod2 in HEK293T human embryonic kidney cells transfected with control small interfering RNA (siRNA), whereas transfection with Pellino3-specific siRNA diminished the reporter activity (Supplementary Fig. 1a,b). Coexpression of either Pellino3s (Supplementary Fig. 1c) or Pellino3l (Supplementary Fig. 1d)

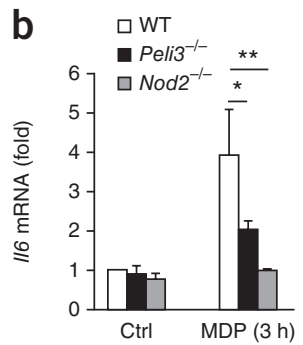

Figure 1 Pellino3 deficiency diminishes MDP-induced expression of proinflammatory cytokines in BMDMs. (a) Enzyme-linked immunosorbent assay (ELISA) of IL-6, IL-1 $\beta$, TNF, IL-12p40 and CXCL1 in medium from wild-type (WT), Peli3 ${ }^{-/-}$and Nod2-I- BMDMs left unstimulated (Ctrl) or treated with MDP $(50 \mu \mathrm{g} / \mathrm{ml})$ for $24 \mathrm{~h}$. (b) Quantitative PCR analysis of $/ / 6, \| 1 b, T n f, / / 12 b$ and $C x C / 1$ mRNA in wild-type, Peli3-l- and Nod2-I- BMDMs left unstimulated or treated with MDP $(50 \mu \mathrm{g} / \mathrm{ml})$ for $3 \mathrm{~h}$; results are presented relative to those of unstimulated wild-type BMDMs, set as 1. (c) Quantitative PCR analysis of mRNA encoding IL-12p40 (IL12b mRNA) in wild-type and $P$ Peli3-/- BMDMs left unstimulated or treated for $2 \mathrm{~h}$ with LPS ( $1 \mu \mathrm{g} / \mathrm{ml}$; top) or for $5 \mathrm{~h}$ with $\mathrm{Pam}_{3} \mathrm{CSK}_{4}$ $(1 \mu \mathrm{g} / \mathrm{ml}$; bottom) in the presence (+MDP) or absence of MDP $(10 \mu \mathrm{g} / \mathrm{ml})$. (d) Immunoblot analysis of phosphorylated ( $\mathrm{p}-$ ) and total I $\mathrm{\kappa B} \alpha, \mathrm{p} 38$, Jnk and Erk in lysates of wild-type and Peli3-/- BMDMs stimulated for 0-120 min (above lanes) with MDP $(50 \mu \mathrm{g} / \mathrm{ml})$; $\beta$-actin serves as a loading control throughout. NS, not significant; ${ }^{*} P<0.05,{ }^{*} P<0.01$ and ${ }^{* * *} P<0.001$ (two-tailed Student's $t$-test). Data are from three independent experiments (mean and s.e.m. in a-c).
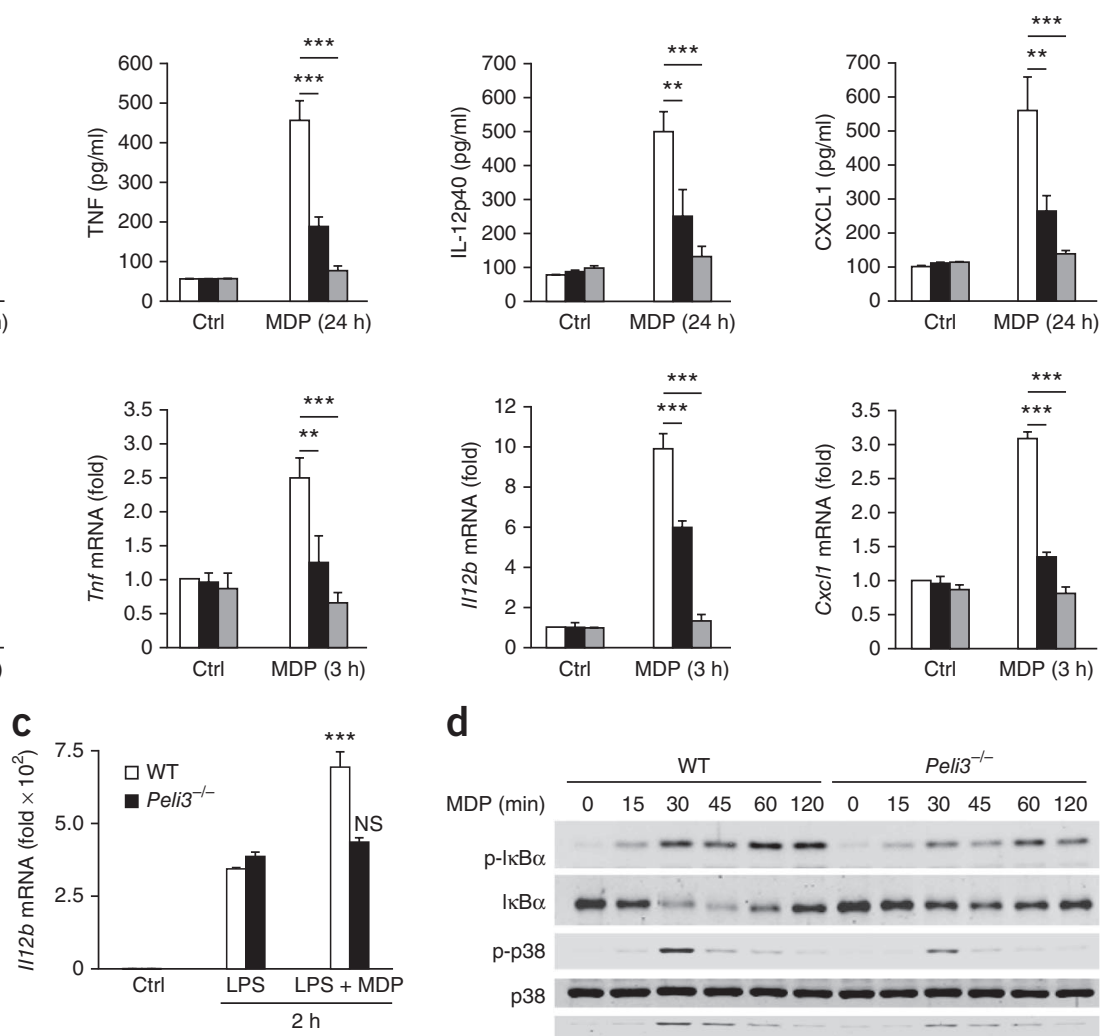

d
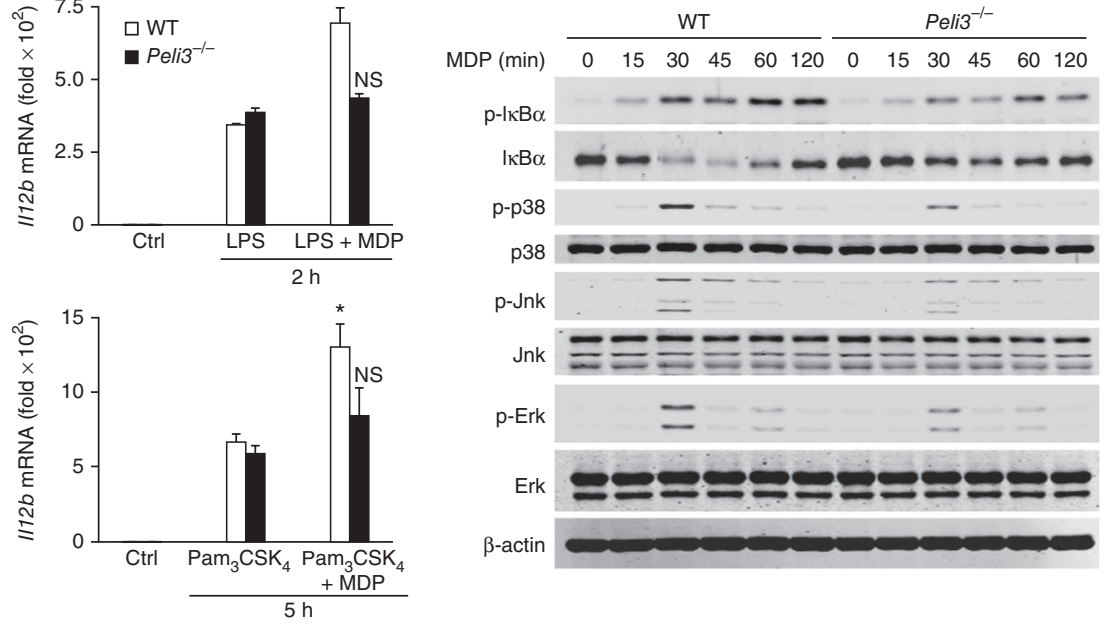
Figure 2 Peli3 $^{-1-}$ mice have less induction of the expression of proinflammatory cytokines in response to MDP. Immunoassay of the expression of IL-6, CXCL1, IL-12p40, IL-1 $\beta$, IL-10 and TNF in serum from wild-type and Peli3-l- mice ( $n=6$ per group) $4 \mathrm{~h}$ after intraperitoneal injection of MDP (25 mg per $\mathrm{kg}$ body weight) or endotoxinfree PBS. Each symbol represents an individual mouse; small horizontal lines indicate the mean (and s.e.m.). ${ }^{*} P<0.05,{ }^{* *} P<0.01$ and $* * * P<0.001$ (two-tailed Student's $t$-test). Data are from three independent experiments.

enhanced Nod2-mediated activation of NF- $\kappa$ B in HEK292T cells. These results indicated a mediatory role for Pellino3 in the Nod2 pathway in both mice and humans.

Because Nod2 is expressed in intestinal epithelial cells as well as myelomonocytic cells, we assessed the role of Pellino3 as a mediator of Nod2 signaling in the intestine. MDP-induced expression of the proinflammatory cytokines IL-6, CXCL1 and CCL2 was much lower in colon explants from Peli3 ${ }^{-/-}$mice than in their wild-type counterparts (Supplementary Fig. 2a). In addition, MDP-induced cytokine expression and phosphorylation of $\mathrm{I} \kappa \mathrm{B} \alpha$, p38 and Jnk was lower in primary intestinal epithelial cells from $\mathrm{Peli}^{-/-}$mice than in their wildtype counterparts (Supplementary Fig. 2b, c). The siRNA-mediated suppression of Pellino3 in the human colonic epithelial cell line HCT116 diminished the ability of MDP to induce the cytokines IL-8, CXCL1 and TNF (Supplementary Fig. 2d) and resulted in much less MDP-induced phosphorylation of I $\mathrm{KB} \alpha$, p38 and Jnk (Supplementary Fig. 2e), which indicated that Pellino3 mediated Nod2 signaling in intestinal epithelial cells.

Given that Nod1 triggered those pathways similarly, we assessed the role of Pellino3 in Nod1 signaling in macrophages and epithelial cells. Suppression of Pellino3 expression by short hairpin RNA (shRNA) in the human monocytic cell line THP-1 inhibited the ability of the Nod1 ligand diaminopimelic acid to trigger phosphorylation of $\mathrm{I} \kappa \mathrm{B} \alpha$, p38 and Jnk (Supplementary Fig. 3a) and to induce IL-8, CXCL1 and TNF (Supplementary Fig. 3b). Similarly, the shRNA-mediated decrease in Pellino3 expression in the human colonic epithelial cell line HT29 suppressed the diaminopimelic acid-induced activation of the NF- $\kappa$ B and MAPK pathways (Supplementary Fig. 3c) and induction of the proinflammatory cytokines noted above (Supplementary Fig. 3d), which indicated that Pellino3 also mediated Nod1 signaling in myelomonocytic cells and intestinal epithelial cells.

Intraperitoneal injection of MDP resulted in higher concentrations of IL-6, CXCL1, IL-12p40, IL-1 $\beta$, IL-10 and TNF in serum from wildtype mice (Fig. 2). Peli3 ${ }^{-1-}$ mice had basal serum concentrations of cytokines similar to those of their wild-type counterparts, but the MDP-induced increase in serum cytokines was significantly diminished. Together these results suggested that Pellino3 was a mediator in the Nod pathway in myelomonocytic and intestinal epithelial cells.

Pellino3 deficiency impairs clearance of Citrobacter rodentium The regulatory function of Nod 2 in intestinal homeostasis ${ }^{3}$ prompted us to evaluate the in vivo role of Pellino3 in controlling inflammation of the intestine. In a model of colitis induced by Citrobacter rodentium, in which Nod2 is central for bacterial clearance ${ }^{40}$, oral gavage with a bioluminescent bacteria resulted in an early peak of bacterial colonization at day 6 after infection in Peli3 ${ }^{-1-}$ mice, whereas wildtype mice reached a peak of infection at day 9 after infection (Fig. 3a). Peli3 $^{-1-}$ mice sustained a high fecal bacterial load from day 6 until day 16 after infection, whereas the peak bacterial load fell sharply in wild-type mice, which resulted in higher fecal bacterial loads in Peli3 $^{-/-}$mice than in wild-type mice at days 12 and 13 after infection. Live bioluminescence imaging showed a slightly more intense
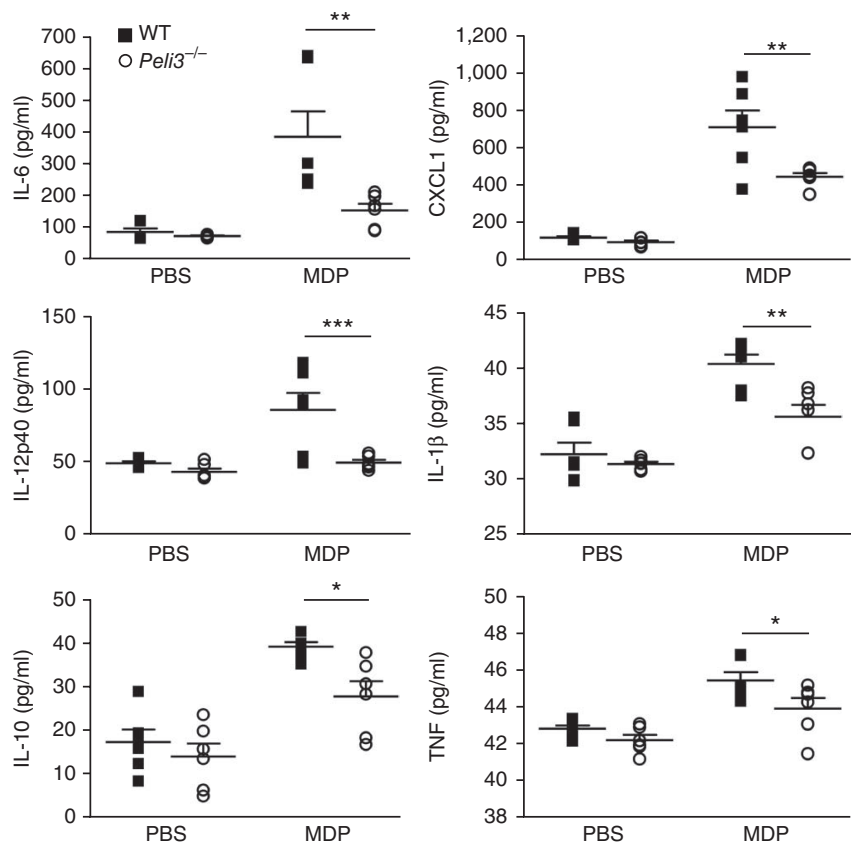

signal in the lower abdomen of infected $\mathrm{Peli3}^{-/-}$mice than in that of infected wild-type mice (data not shown), a result we confirmed by the significantly higher bioluminescent signal in infected $\mathrm{Peli3}^{-/-}$ colons than in wild-type colons at day 12 after infection (Fig. 3b). Peli3 ${ }^{-1-}$ mice had a higher fecal bacterial load on day 19 than did wild-type mice (Fig. 3a), which suggested a delay in bacterial clearance. After infection, C. rodentium did not spread to other mucosal or systemic organs in wild-type or Peli3 ${ }^{-/-}$mice. As reported before ${ }^{40,41}$, we found that infected wild-type mice had significantly greater colon weight and shorter colons at day 12 after infection than before infection (Fig. 3c). Despite their higher bacterial load at that time point, infected Peli3-/- mice did not develop those signs of inflammation (Fig. 3a,c). The histological scores of distal colons at day 12 after infection indicated that $\mathrm{Peli3}^{-/-}$mice had less mucosal thickening (leukocyte infiltration), shorter colonic crypts (Fig. 3d) and less goblet-cell depletion (Fig. 3e) than did their wild-type counterparts. Analysis of proinflammatory cytokines in distal colon homogenates showed less IL-1 $\beta$, CCL2, IL- 6 and CXCL1 in Peli3 ${ }^{-1-}$ mice than in wild-type mice on day 12 after infection (Fig. 3f). We found no major difference between the two groups in the concentration of TNF, IL-10, IL-12p70 or interferon- $\gamma($ IFN- $\gamma)$. Phenotyping of cells of the immune system from isolated mesenteric lymph nodes showed that Peli3-/mice had a significantly fewer monocytes $\left(\mathrm{CD} 11 \mathrm{~b}^{+} \mathrm{F} 4 / 80^{+} \mathrm{Gr}-1^{+}\right)$, $\mathrm{CD}^{+}{ }^{+} \mathrm{CD} 4^{+} \mathrm{IL}-17^{+} \mathrm{T}$ cells and $\mathrm{CD} 4^{+} \mathrm{IFN}-\gamma^{+} \mathrm{T}$ cells than did wildtype mice at day 12 after infection (Fig. 3g). However, in correlation with the greater fecal bacterial loads of $\mathrm{Peli3}^{-/-}$mice at later time points during infection, on day 21 after infection, the distal colons of Peli3 $^{-1-}$ mice had a higher histological inflammatory score and a higher score for staining with periodic acid Schiff than did those of wild-type mice (Fig. 3e,h), which suggested a delay in the recovery of goblet cells and mucus production in Peli3 ${ }^{-1-}$ mice. Thus, loss of Pellino3 resulted in less early inflammation and impaired clearance of $C$. rodentium. Because Nod2 mediates intestinal clearance of C. rodentium by promoting the production of inflammatory cytokines, the recruitment of monocytes and enhanced responses of the $\mathrm{T}_{\mathrm{H}} 1$ and $\mathrm{T}_{\mathrm{H}} 17$ subsets of helper $\mathrm{T}$ cells in the intestine ${ }^{40}$, these findings were consistent with a role for Pellino3 in mediating the protective effects of Nod2 in this infection model. 

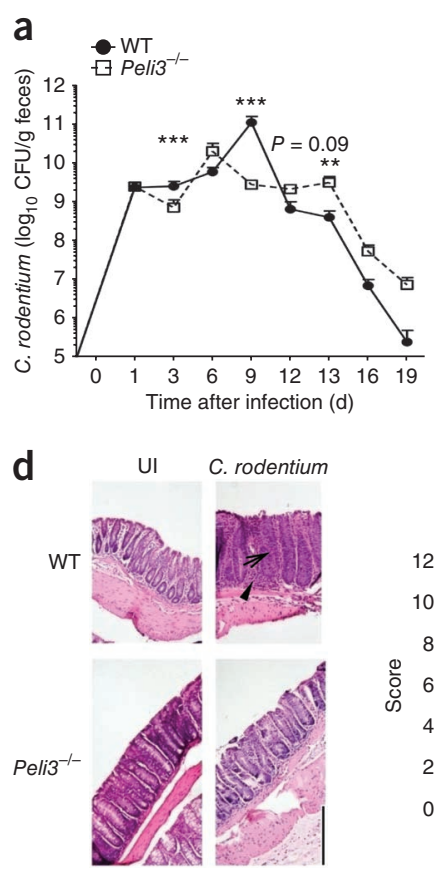

f

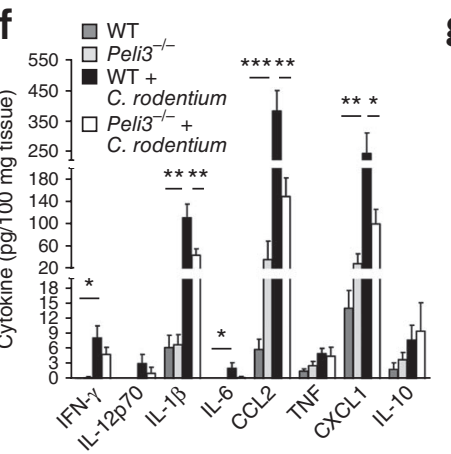

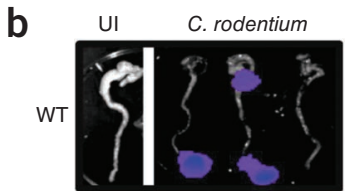

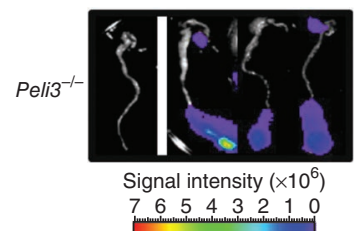

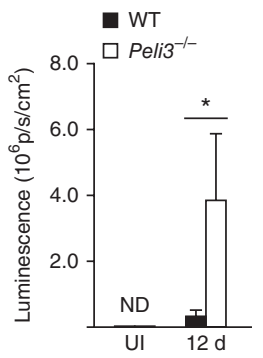

C

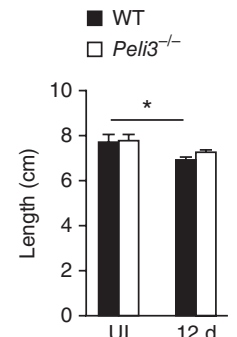

WT

$\square$ Peli3 $^{-1-}$

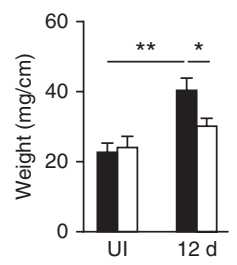

e

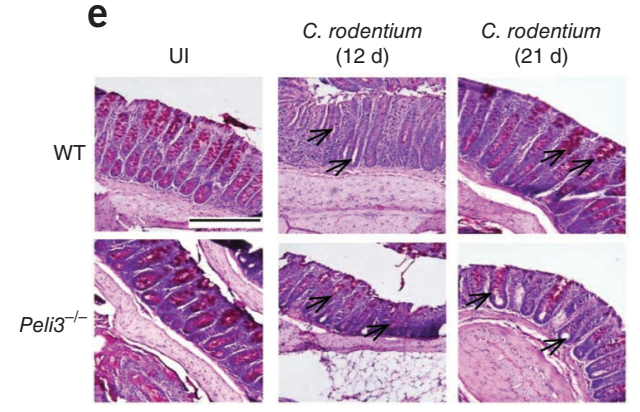

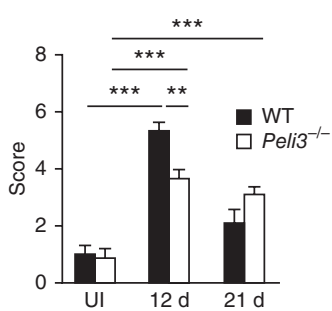

h

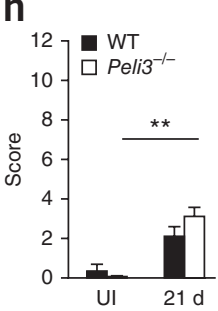

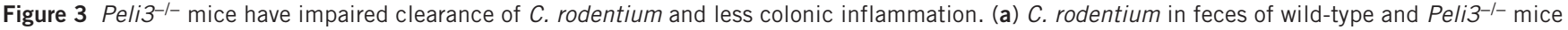
( $n=8-17$ per time point) on days 0-19 (horizontal axis) after infection with $C$. rodentium, presented as $\log _{10}$ colony-forming units (CFU) per gram feces. (b) Bioluminescent images (left) of the colon and cecum of uninfected (UI) and C. rodentium-infected wild-type and Peli3-l- mice $(n=8-9$ per group) at day 12 after infection (red, most intense light emission; blue, weakest signal), and quantification of that luminescence (right). (c) Colon length and weight of uninfected and $C$. rodentium-infected wild type and Peli3 ${ }^{-1-}$ mice ( $n=4$ per group (uninfected) or 9 per group (infected)) at day 12 after infection. (d) Hematoxylin-and-eosin staining of distal colon sections from uninfected and $C$. rodentium-infected wild-type and Peli3-I- mice on day 12 after infection (left), and histological inflammatory scores of such sections (right; $n=4$ mice per group (uninfected) or 8-9 mice per group (infected)). Arrowhead (left) indicates leukocyte infiltration; arrow indicates crypt elongation. Scale bar, $200 \mu \mathrm{m}$. (e) Periodic acid Schiff staining of sections of distal colons from uninfected and $C$. rodentium-infected wild-type and Peli3-I- mice on days 12 and 21 after infection (left), and histological scores of such sections (right; $n=3-4$ mice per group (uninfected) or 8-9 mice per group (infected)). Arrows (left) indicate differences in mucin staining in goblet cells. Scale bar, $200 \mu \mathrm{m}$. (f) Cytokines in colons from uninfected and C. rodentium-infected wild-type and Peli3-l- mice ( $n=3-4$ per group (uninfected) or 8-9 per group (infected)). (g) Frequency of monocytes (CD1 1 b $\left.\mathrm{b}^{+} \mathrm{F} / 80^{+} \mathrm{Gr}-1^{+}\right)$and IL-17- or IFN- $\gamma$-producing CD4+ ${ }^{+}$cells in mesenteric lymph nodes from uninfected and C. rodentium-infected wild-type and Peli3 ${ }^{-1-}$ mice ( $n=3-4$ per group (uninfected) or 7-9 per group (infected)) on day 12 after infection. Each symbol represents an individual mouse; small horizontal lines indicate the mean (and s.e.m.). (h) Histological inflammatory scores of distal colonic sections from uninfected and $C$. rodentium-infected wild-type and Peli3-l- mice ( $n=3$ per group (uninfected) or 8 per group (infected)) on day 21 after infection. ND, not detected. ${ }^{*} P<0.05,{ }^{*} P<0.01$ and ${ }^{* * *} P<0.001$ (Mann-Whitney $U$-test (a,b), one-way analysis of variance followed by Bonferroni's multiple-comparison test $(\mathbf{c}-\mathbf{f}, \mathbf{h})$ or unpaired $t$-test with Welch's correction $(\mathbf{g}))$. Data are from one experiment.

Pellino3 is protective in chemically induced models of colitis We next assessed whether Pellino3 was protective in two chemically induced models of colitis (induction with dextran sodium sulfate (DSS) or 2,4,6-trinitro benzene sulfonic acid (TNBS)) in which Nod2 has a demonstrated protective role ${ }^{42}$. We provided mice with $2.5 \%$ DSS in the drinking water for $5 \mathrm{~d}$ and injected them intraperitoneally with MDP or endotoxin-free PBS on days 0, 1 and 2 and monitored their morbidity and body weight each day. DSS-treated wild-type mice developed progressive weight loss starting at day 4 , whereas the administration of MDP diminished the severity of the weight loss (Supplementary Fig. 4a). As reported before ${ }^{42}$, the DSS-induced weight loss was not ameliorated by the administration of MDP to Nod $2^{-/-}$mice (Supplementary Fig. 4a). DSS-treated Peli3 ${ }^{-/-}$mice developed weight loss similar to that of wild-type mice, but administration of MDP did not ameliorate this. Treatment with MDP resulted in less DSS-induced colon shortening in wild-type mice but not in Nod $2^{-/-}$or Peli3 ${ }^{-/-}$mice (Supplementary Fig. 4b). Histological analysis of sections of distal colons from untreated wild-type and $\mathrm{Peli3}^{-1-}$ 
a
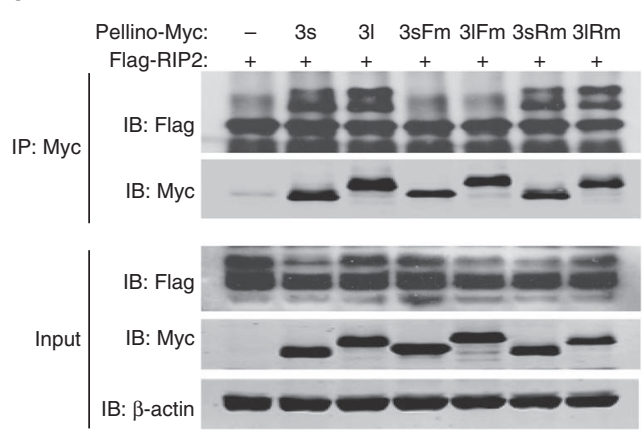

b

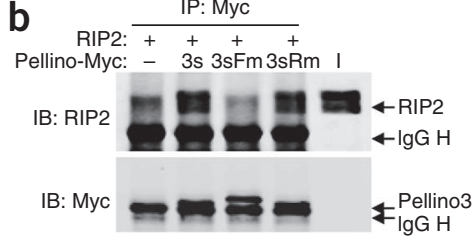

d

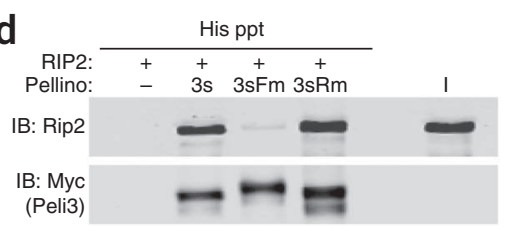

C

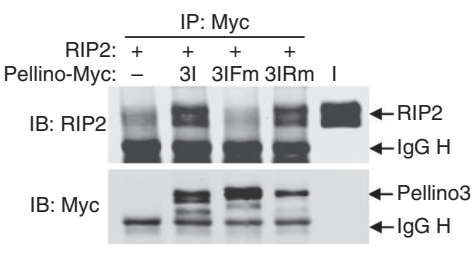

e

$\begin{array}{cccccc}\text { MDP (min) } & 0 & 15 & 30 & 70 & 30\end{array}$

IB: Pellino3 a k :

IB: RIP2 $\approx-1=12$ \&IPGH

Input $\mid \begin{array}{ll}\text { IB: Pellino3 } \\ \text { IB: RIP2 }\end{array}$

Figure 4 Pellino3 interacts directly with RIP2 in a FHA domain-dependent manner. (a) Immunoassay of RIP2 and Pellino3 in HEK293T cells transfected with constructs encoding Flag-tagged RIP2 (Flag-RIP2 +) alone (-) or with Myc-tagged (Pellino-Myc) Pellino3s (3s) or Pellino3I (3I) or the corresponding mutants with a nonfunctional FHA domain (3sFm or 3IFm) or an altered RING domain (3sRm or 3IRm), followed by immunoprecipitation (IP) with antibody to Myc (anti-Myc) and immunoblot analysis (IB), with anti-Flag (RIP2) or anti-Myc (Pellino3), of immunoprecipitates or of lysates without immunoprecipitation (Input). (b,c) Immunoassay of RIP2 and Pellino3 in HEK293T cells transfected with a construct encoding Flag-tagged RIP2 and incubated alone (-) or with Myc-tagged purified recombinant forms (as in a) of Pellino3s (b) or Pellino3I (c), followed by immunoprecipitation with anti-Myc and immunoblot analysis of immunoprecipitates (left and middle) or of input without immunoprecipitation (I; far right). (d) Immunoblot analysis of RIP2 and Pellino among proteins precipitated with nickel agarose (His ppt) from samples of purified recombinant RIP2 incubated alone (-) or in the presence of purified recombinant forms (as in a) of Myc- and histidine-tagged Pellino3s, or of input without precipitation (I). (e) Immunoassay of Pellino3 and RIP2 in HEK293T cells transfected with an expression plasmid encoding Nod2 and then stimulated for 0-70 min (above lanes) with $\operatorname{MDP}(50 \mu \mathrm{g} / \mathrm{ml})$, followed by immunoprecipitation with anti-RIP2 or isotype-matched control antibody (immunoglobulin G (IgG); far right (C)) and immunoblot analysis of immunoprecipitates or lysates with anti-Pellino3 or anti-RIP2. Arrows indicate the migration of Pellino3, RIP2 and the IgG heavy chain (IgGH). Data are representative of three experiments.

mice showed an intact intestinal epithelium (Supplementary Fig. 4c). After treatment with DSS, the distal colons of wild-type mice had considerable infiltration of inflammatory cells, severe lesions of the intestinal epithelium and loss of goblet cells, which was ameliorated by the administration of MDP (Supplementary Fig. 4c). In contrast, DSS-treated Peli3 ${ }^{-/-}$mice were not protected from colitis pathology after administration of MDP (Supplementary Fig. 4d). These results were consistent with loss of the protective effects of MDP on colitis severity in Nod2 $2^{-/-}$and Peli3 ${ }^{-/-}$mice. In the TNBS-induced model, $\mathrm{Peli3}^{-/-}$mice developed greater weight loss, more colon shortening, greater exacerbation of tissue damage and worse overall pathology than did their wild-type littermates in response to the administration of TNBS (Supplementary Fig. 5a-d). These results indicated a role for Pellino3 in intestinal homeostasis and in mediating the protective effects of Nod2 in colitis.

\section{Pellino3 interacts with RIP2}

To probe the mechanistic basis of the mediatory role of Pellino3 in the Nod2 pathway, we initially assessed the ability of Pellino3 to interact with RIP2. RIP2 immunoprecipitated together with both Pellino3s and Pellino3l in HEK293 cells coexpressing RIP2 and either spliced form of Pellino3 (Fig. 4a). RIP2 did not immunoprecipitate together with mutant forms of Pellino3s or Pellino3l with a nonfunctional FHA domain, whereas alteration of the Pellino3 RING-like domain had no effect on the Pellino3-RIP2 interaction (Fig. 4a). Purified recombinant Pellino3s and Pellino3l, as well as the mutant forms with an altered RING-like domain, immunoprecipitated together with RIP2 from cell lysates, but the mutant forms with a nonfunctional FHA domain did not (Fig. 4b,c). Furthermore, purified recombinant Pellino3s and Pellino3s with an altered RING-like domain immunoprecipitated abundantly together with purified RIP2, but Pellino3s with a nonfunctional FHA domain did not (Fig. 4d). Finally, stimulation of Nod2 signaling in HEK293T cells by MDP promoted a time-dependent interaction beween endogenous Pellino3 and RIP2 (Fig. 4e). These results indicated that Pellino3 directly interacted with RIP2 via its FHA domain.

\section{Pellino3 ubiquitinates RIP2}

We next assessed the ability of Pellino3 to regulate the ubiquitination of RIP2. Overexpression of Pellino3s or Pellino3l with RIP2 in HEK293 cells resulted in substantial polyubiquitination of RIP2 (Fig. 5a). Coexpression of A20, an enzyme known to counter-regulate Nod2 signaling by deubiquitinating RIP2 (ref. 43), abrogated the Pellino3-mediated ubiquitination of RIP2 (Fig. 5a). Because RIP2mediated signaling is dependent on modification with K63-linked polyubiquitin chains, we assessed the type of linkage induced by Pellino3 in the polyubiquitination of RIP2. We expressed Pellino3 with RIP2 and various forms of ubiquitin, ranging from wild-type ubiquitin containing all seven of its lysine residues to mutants lacking all lysine residues or containing a single lysine at various positions (Fig. $\mathbf{5 b}$ ). Pellino3s promoted ubiquitination of RIP2 when coexpressed with wild-type ubiquitin or mutant ubiquitin with a single lysine at position 63. With that ubiquitin mutant, wild-type Pellino3s and Pellino3l were equally able to promote K63-linked polyubiquitination of RIP2, whereas Pellino3s and Pellino3l mutants with alteration of the RING domain or FHA domain were totally ineffective (Fig. 5c). Wild-type Pellino3s and Pellino3l and their mutants with alteration of the RING domain or FHA domain did not ubiquitinate RIP2 when coexpressed with ubiquitin with a single point substitution of arginine for lysine at position 63 (K63R ubiquitin; Fig. 5d), whereas wild-type Pellino3s and Pellino3l promoted ubiquitination of RIP2 when expressed with K48R ubiquitin, but the mutant forms of Pellino3s and Pellino3l did not (Fig. 5e). This showed that Pellino3 ubiquitinated RIP2 exclusively via K63 linkages.

We next used an in vitro ubiquitination assay with purified Pellino3s and recombinant RIP2 to assess whether Pellino3 directly ubiquitinated RIP2. Pellino3 catalyzed polyubiquitination of RIP2 in the presence of recombinant mutant ubiquitin with a single lysine 


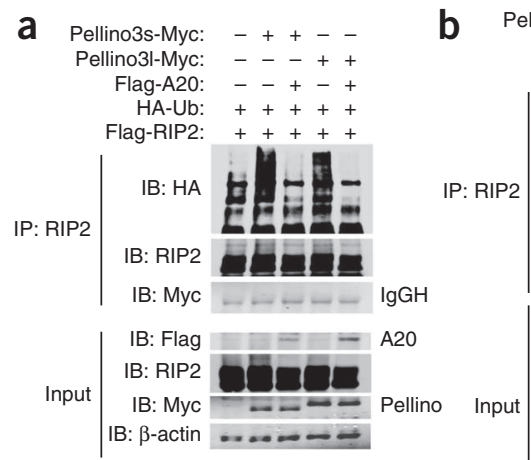

d
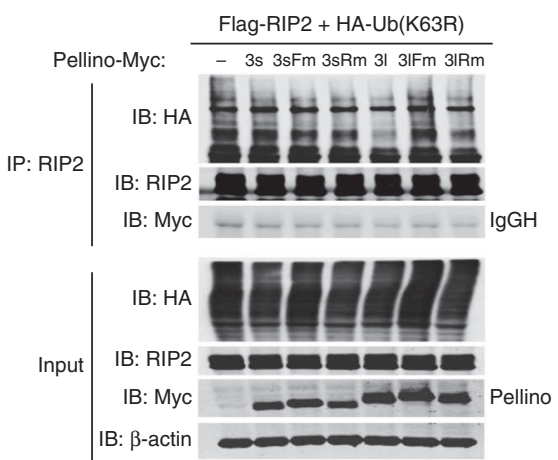

Pellino3s-Myc: -+-+-+-+-+-+-+-+-+ Flag-RIP2: $++++++++++++++++++\quad+++$ HA-Ub: $\overline{\text { WT }} \overline{\mathrm{K} 0} \overline{\mathrm{K} 6} \overline{\mathrm{K} 11} \overline{\mathrm{K} 27} \overline{\mathrm{K} 29} \overline{\mathrm{K} 33} \overline{\mathrm{K} 48} \overline{\mathrm{K} 63} \quad \overline{\mathrm{WT}}$

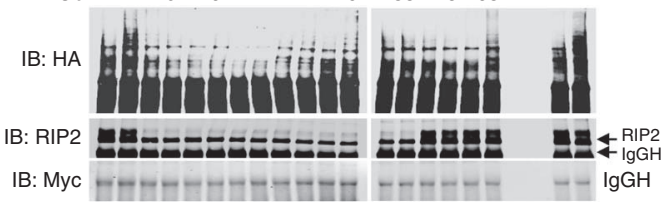

C

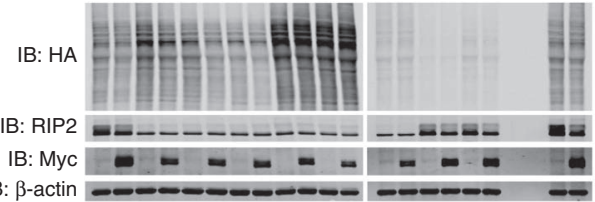

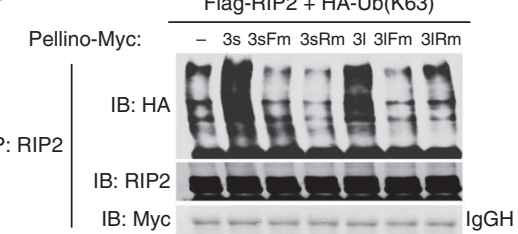

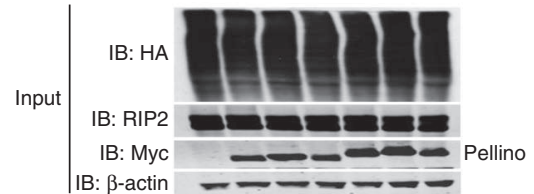

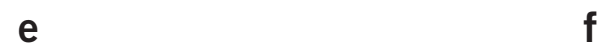

e
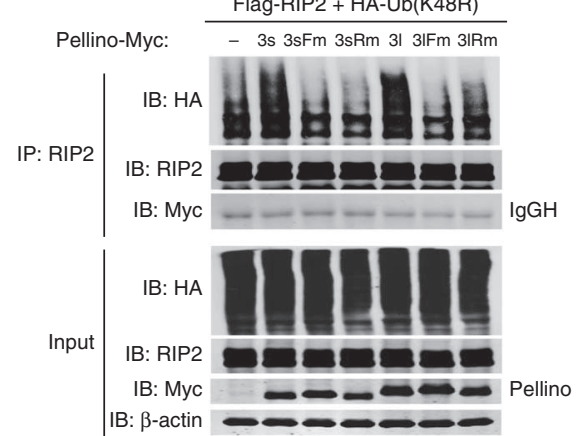

f

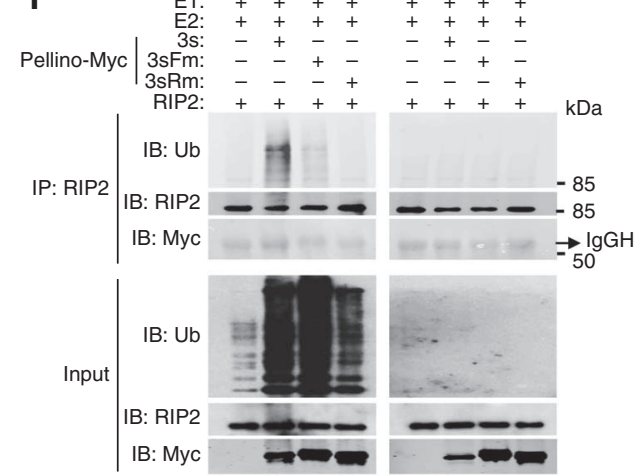

Figure 5 Pellino3 catalyzes K63-linked polyubiquitination of RIP2. (a-e) Immunoassay of ubiquitin, RIP2 and Pellino3 in HEK293T cells transfected with constructs encoding various combinations (above lanes) of Flag-tagged RIP2 (a-e) and Myc-tagged Pellino3s or Pellino3I, Flag-tagged A20 (Flag-A20) and hemagglutinin (HA)-tagged wild-type ubiquitin (HA-Ub) (a), Myc-tagged Pellino3s and HA-tagged wild-type ubiquitin or mutant ubiquitin lacking all seven lysine residues (K0) or with substitution of alanine for all lysine residues except the residue noted (K6, K11, K27, K29, K33, K48, K63) (b), HA-tagged mutant ubiquitin with substitution of alanine for all lysine residues except K63 (HA-Ub(K63)) and Myc-tagged Pellino3s or Pellino3I (wild-type and mutants as in Fig. 4a) (c), HA-tagged mutant ubiquitin with the K63R substitution (HA-Ub(K63R)) and Myc-tagged Pellino3s or Pellino3I as in c (d) or HA-tagged ubiquitin with the K48R substitution (HA-Ub(K48R)) and Myc-tagged Pellino3s or Pellino3I as in c (e), followed by immunoprecipitation with anti-RIP2 and immunoblot analysis of immunoprecipitates or lysates with anti-HA, anti-RIP2 or anti-Myc. (f) In vitro ubiquitination assay of E1 (100 nM), UbcH13-Uev1a (E2; $500 \mathrm{nM})$, purified recombinant RIP2 (400 ng) and ubiquitin (4 $\mu \mathrm{g})$ with a single lysine at position 63 (left) or with the K63R substitution (right), in the presence (+) or absence (-) of recombinant forms (as in c) of Myc-tagged Pellino3s $(0.5 \mu \mathrm{g})$, followed by immunoprecipitation with anti-RIP2 and immunoblot analysis of immunoprecipitates or lysates with anti-ubiquitin (Ub), anti-RIP2 or anti-Myc. Data are representative of three independent expertiments.

at position 63 but not in the presence of K63R ubiquitin (Fig. 5f). Alterations in the RING and FHA domains of Pellino3 abrogated the in vitro ubiquitination of RIP2. Pellino3s with a nonfunctional FHA domain retained its E3 ligase catalytic activity, as it induced the formation of unanchored polyubiquitin chains, but it was unable to ubiquitinate RIP2, probably because of its inability to interact with RIP2 (Fig. 5f).

We next probed the structural features of RIP2 that facilitated the Pellino3-RIP2 association. RIP2 has an amino-terminal kinase domain and a carboxy-terminal CARD that are linked by an intermediate domain (Supplementary Fig. 6a). A truncated form of RIP2 that still contained the kinase domain immunoprecipitated with Pellino3 (Supplementary Fig. 6b), whereas the intermediate domain and CARD were insufficient for binding to Pellino3 (Supplementary Fig. $\mathbf{6 b}, \mathbf{c}$ ). However, the kinase activity of RIP2 was not required for its interaction with Pellino3 or ubiquitination, as a kinase-inactive form of RIP2 (with substitution of arginine for the lysine at position 47) ${ }^{44}$ interacted with Pellino3 and was ubiquitinated to the same extent as wild-type RIP2 (Supplementary Fig. 6d,e). Because ubiquitination of RIP2 at Lys209, in its kinase domain, is important for downstream activation of NF- $\kappa B^{16}$, we assessed if Pellino3 promoted ubiquitination of RIP2 at Lys209. A mutant form of RIP2 with substitution of arginine for the lysine at position 209 immunoprecipitated together with Pellino3 and still underwent Pellino3-mediated ubiquitination by K63-linked polyubiquitin chains (Supplementary Fig. 6f,g). Thus Pellino3 promoted K63-linked polyubiquitination of RIP2, at a site (or sites) other than Lys209, and in an FHA- and RING-like domaindependent manner.

\section{Pellino3 mediates Nod2-induced polyubiquitination of RIP2}

We next assessed the physiological relevance of the Pellino3-mediated polyubiquitination of RIP2 in the Nod2 pathway. MDP stimulated the polyubiquitination of endogenous RIP2 in a time-dependent manner in wild-type BMDMs, but this was much lower in Peli3-/- BMDMs (Fig. 6a). Through the use of antibodies that selectively recognize K63-linked or K48-linked polyubiquitin chains, we found MDPinduced K63 polyubiquitination of endogenous RIP2 in wild-type BMDMs (Fig. 6a,b). MDP did not promote detectable K63-linked ubiquitination of RIP2 in Peli3 ${ }^{-/-}$BMDMs.

Because K63-linked ubiquitination of RIP2 is associated with corecruitment of the TAK1 and IKK complexes into close proximity, which leads to downstream activation of NF- $\kappa B$, we assessed the effects of Pellino3 deficiency on the Nod2-induced interaction of RIP2 with TAK1 and IKK. MDP promoted substantial and 


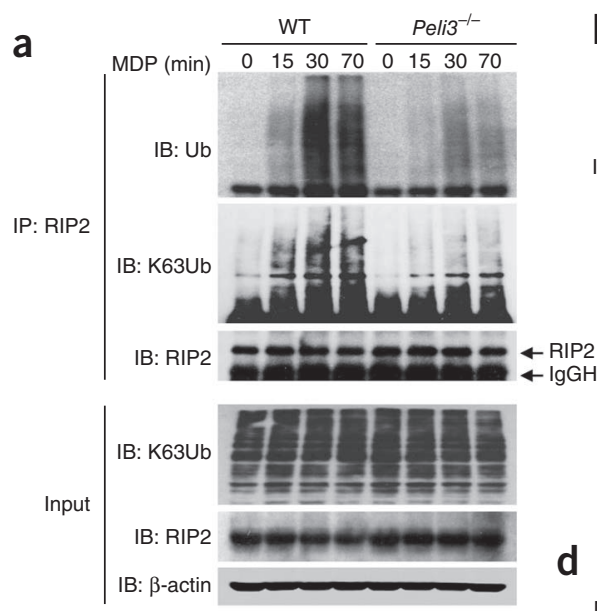

Figure 6 Pellino3 mediates MDPinduced K63-linked polyubiquitination of RIP2 and downstream signaling in an IAP-independent manner. (a-c) Immunoassay of wild-type and Peli3-/- BMDMs stimulated for 0-70 min (above lanes) with $\operatorname{MDP}(50 \mu \mathrm{g} / \mathrm{ml})$, followed by immunoprecipitation with anti-RIP2 and immunoblot analysis of immunoprecipitates or lysates with anti-RIP2 (a-c) and antibody to ubiquitin (Ub) or to K63-linked ubiquitin (K63Ub) (a), antibody to K48-linked ubiquitin (K48Ub) (b) or anti-TAK1, anti-IKK $\gamma$ and anti-Sharpin (c). (d,e) Immunoassay of wild-type and Peli3 ${ }^{-1}$ BMDMs given no pretreatment (-) or pretreated (+) for 30 min with BV6 $(20 \mu \mathrm{M})(\mathrm{d})$ or for $1 \mathrm{~h}$ with AT406 $(30 \mu \mathrm{M})(\mathbf{e})$ and then stimulated for 0-70 min (above lanes) with MDP (50 $\mu \mathrm{g} / \mathrm{ml})$, followed by immunoprecipitation with anti-RIP2 and immunoblot analysis of immunoprecipitates with antibody to ubiquitin or to K63-linked ubiquitin and anti-RIP2 and immunoblot analysis of lysates with antibody to all clAP proteins (Pan-clAP), anti-XIAP, anti-RIP2 and antibodies to phosphorylated ( $\mathrm{p}-$ ) and total I $\mathrm{kB} \alpha$ and $\mathrm{p} 38$. Right margin (c), molecular size in kilodaltons (kDa); arrows (right margins, a-e) indicate the migration of RIP2 and the IgG heavy chain (IgGH). Data are representative of three independent experiments. time-dependent recruitment of TAK1 and IKK $\gamma(\mathrm{NEMO})$ to RIP2 in wild-type BMDMs, but this was abrogated in Peli3 ${ }^{-1-}$ BMDMs (Fig. 6c). However, published reports have indicated that IAP proteins regulate the Nod2-induced ubiquitination of RIP2 (refs. 23,24). To assess the relative importance of Pellino3 and the IAP proteins in this pathway and their potential functional interaction, we stimulated BMDMs from wild-type and Peli3 ${ }^{-/}$mice with MDP in the presence or absence of the IAP antagonist BV6, which pharmacologically depletes cells of cIAP1 and cIAP2. Treatment of wild-type cells with BV6 did not affect the ability of MDP to promote the K63-linked ubiquitination of RIP2 or downstream phosphorylation of $\mathrm{I} \kappa \mathrm{B} \alpha$ and p38 (Fig. 6d). The MDPinduced ubiquitination of RIP 2 and phosphorylation of $\mathrm{I} \kappa \mathrm{B} \alpha$ and $\mathrm{p} 38$ were substantially compromised in Peli3 ${ }^{-1-}$ BMDMs, and these effects were not further regulated by the addition of BV6 (Fig. 6d).

Because BV6 does not affect the expression of XIAP, we repeated the experiments described above with AT406, a compound that depletes cells of cIAP1 and cIAP2 and results in less XIAP in BMDMs. AT406 partially diminished the MDP-induced total ubiquitination of RIP2 in wild-type BMDMs, especially at later time points, but did not affect the MDP-induced K63-linked ubiquitination of RIP2 (Fig. 6e). AT406 also had a slight inhibitory effect on the MDP-induced phosphorylation of $I \kappa \mathrm{B} \alpha$ and p38 in wild-type BMDMs, which was weaker than the effects of Pellino3 deficiency in Peli3 ${ }^{-1-}$ BMDMs (Fig. 6e). However, treatment of Peli3-/- BMDMs with AT406 further augmented the inhibitory effects associated with Pellino3 deficiency (Fig. 6e), which suggested that Pellino3 and XIAP may work in parallel. Because XIAP functions in Nod2 signaling by promoting recruitment of the LUBAC ${ }^{23}$, we investigated the MDP-induced recruitment of the LUBAC component Sharpin to RIP2 in wild-type and Peli3 ${ }^{-/-}$ BMDMs. MDP promoted strong and time-dependent interaction of Sharpin with RIP2 in wild-type BMDMs, and this interaction was preserved in Peli3 ${ }^{-/-}$cells (Fig. 6c). These data suggested a critical role for Pellino3 in the Nod2-induced K63-linked ubiquitination of RIP2 and recruitment of TAK1 and IKK complexes. Our results also suggested that XIAP worked in parallel with Pellino3 and were consistent with a published report demonstrating that XIAP promotes ubiquitination of RIP2, in a non K63-linked manner, which leads to the recruitment of the LUBAC and downstream signaling ${ }^{23}$. Whereas Pellino3 and XIAP seemed to act in parallel and independently of each other, they both fed into the same downstream pathways, given the 
a

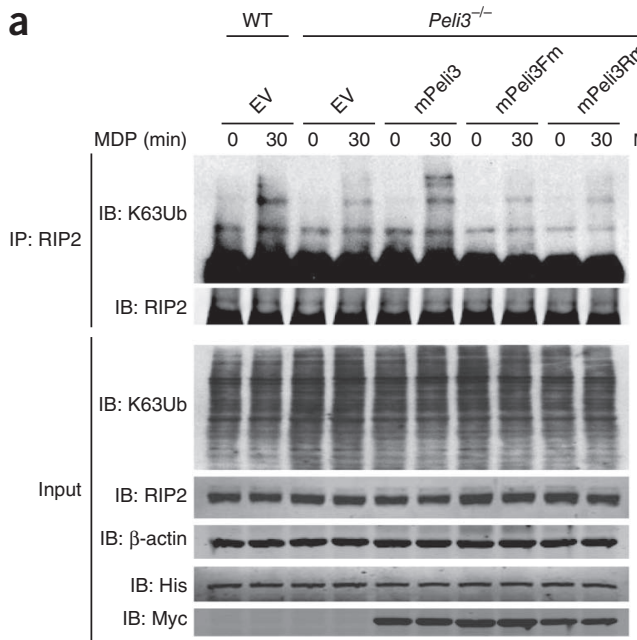

b

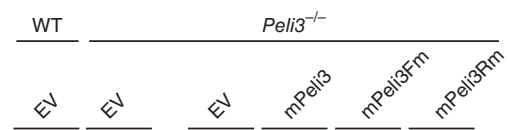

C

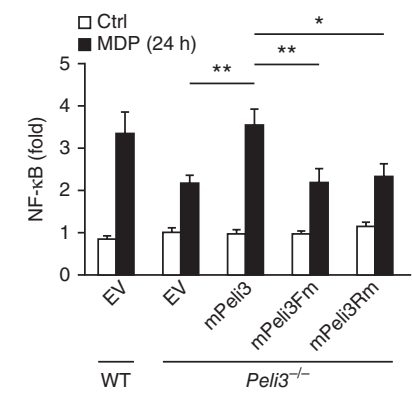

WT Peli3 ${ }^{-1-}$
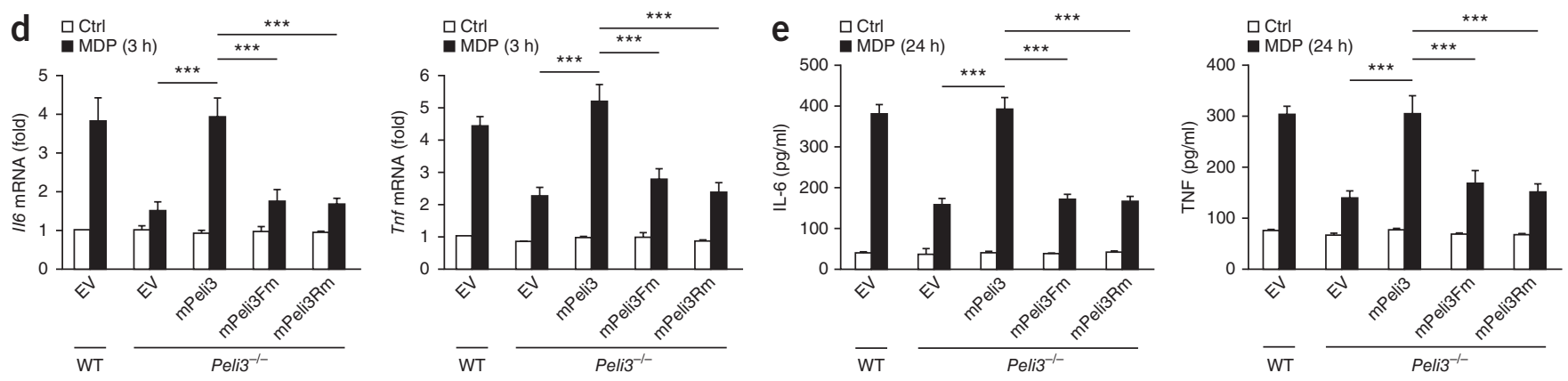

Figure 7 The FHA and RING domains of Pellino3 are both needed to mediate MDP-induced ubiquitination of RIP2 and downstream signaling. $(\mathbf{a}, \mathbf{b})$ Immunoassay of wild-type and Peli3-/- MEFs transfected with an expression construct for histidine-tagged Nod2 and empty vector (EV) or plasmid encoding Myc-tagged wild-type mouse Pellino3 (mPeli3) or mouse Pellino3 with a mutant FHA domain (mPeli3Fm) or RING domain (mPeli3Rm), left untreated or treated with MDP $(50 \mu \mathrm{g} / \mathrm{ml})$, followed by immunoprecipitation with anti-RIP2 and immunoblot analysis of immunoprecipitates with antibody to K63-linked ubiquitin and anti-RIP2 and immunoblot analysis of lysates with those antibodies plus anti-histidine (His) and anti-Myc (a), or immunoblot analysis of lysates with antibodies to phosphorylated ( $\mathrm{p}-$ ) and total I $\mathrm{kB} \alpha, \mathrm{p} 38$, Jnk and Erk, plus anti-histidine and anti-Myc (b). (c) NF-KB activity in wild-type and Peli3-l- MEFs transfected with an expression construct for Nod2 and with empty vector (EV) or plasmid encoding mouse Pellino3 (wild-type or mutant as in a,b), plus an NF-kB-regulated firefly luciferase construct, then left untreated or treated for $3 \mathrm{~h}$ with MDP $(50 \mu \mathrm{g} / \mathrm{ml})$, and assessed as firefly luciferase activity normalized to renilla luciferase activity and presented relative to that untreated wild-type cells transfected with empty vector, set as 1 (top), and immunoblot analysis of Nod2 and Pellino3 in those cells (bottom). (d,e) Quantitative PCR analysis of $1 / 6$ and Tnf mRNA (d) and ELISA of IL-6 and TNF (e) in wild-type and Peli3-/- MEFs transfected with an expression construct for Nod2 and with empty vector or plasmid encoding mouse Pellino3 (wild-type or mutant as in a,b), then left untreated or treated for $24 \mathrm{~h}$ with MDP (50 $\mu \mathrm{g} / \mathrm{ml}$ ); PCR results (d) are presented relative to those of untreated wild-type cells transfected with empty vector, set as $1 .{ }^{*} P<0.05,{ }^{* *} P<0.01$ and ${ }^{* * *} P<0.001$ (two-tailed Student's $t$-test). Data are from three independent experiments (mean and s.e.m. in c-e).

additive nature of the inhibitory effects of the XIAP inhibitor AT406 and Pellino3 deficiency on Nod2 signaling.

\section{Nod2 depends on Pellino3 FHA and RING-like domains}

We next sought to probe the functional relevance of the Pellino3mediated K63-ubiquitination of RIP2 for downstream signaling in the Nod2 pathway. The deficiency in MDP-induced K63-polyubiquitination of RIP2 in Nod2-expressing Peli3 ${ }^{-/-}$mouse embryonic fibroblasts (MEFs) was 'rescued' by reconstitution with wild-type mouse Pellino3, but Pellino3 with an altered RING-like domain or a nonfunctional FHA domain did not 'rescue' that deficiency (Fig. 7a). The loss of MDP-induced phosphorylation of $\mathrm{I} \kappa \mathrm{B} \alpha$ and of $\mathrm{p} 38$, Jnk and Erk in Peli3 $^{-1-}$ MEFs (relative to that in wild-type MEFs) was 'rescued' by the reintroduction of wild-type mouse Pellino3, but not by either of the mutant forms (Fig. 7b). The loss of MDP-induced activation of NF- $\kappa \mathrm{B}$ in Peli3 ${ }^{-/-}$MEFs, as measured by the induction of an NF- $\kappa B$-regulated luciferase reporter gene, was 'rescued' by reconstitution with mouse Pellino3, but not by the Pellino3 mutants with alteration of the FHA or RING-like domain (Fig. 7c). Mouse Pellino3 also restored the MDP-induced expression of the mRNA and protein products of the NF- $\kappa \mathrm{B}$-responsive genes Il6 and Tnf in Peli3 ${ }^{-/-}$MEFs (Fig. 7d,e). The Pellino3 mutants with alteration of the FHA or RINGlike domain were ineffective (Fig. 7d,e). In addition, human Pellino3 was able to reconstitute MDP-induced K63-linked ubiquitination of RIP2, downstream signaling and cytokine expression in THP-1 cells in which Pellino3 expression was ablated by a Pellino3-specific shRNA construct, but Pellino3 mutants with alteration of the FHA or RING-like domain were ineffective (Supplementary Fig. 7a-c). These data indicated that Pellino3-mediated K63-linked ubiquitination of RIP2 was an important step in the Nod2 pathway that leads to downstream activation of NF- $\kappa \mathrm{B}$ and induction of genes encoding proinflammatory molecules.

\section{Lower expression of Pellino3 in Crohn's disease}

Because Pellino3 mediated the protective effects of Nod2 in experimental colitis, we assessed Pellino3 expression in patients with 

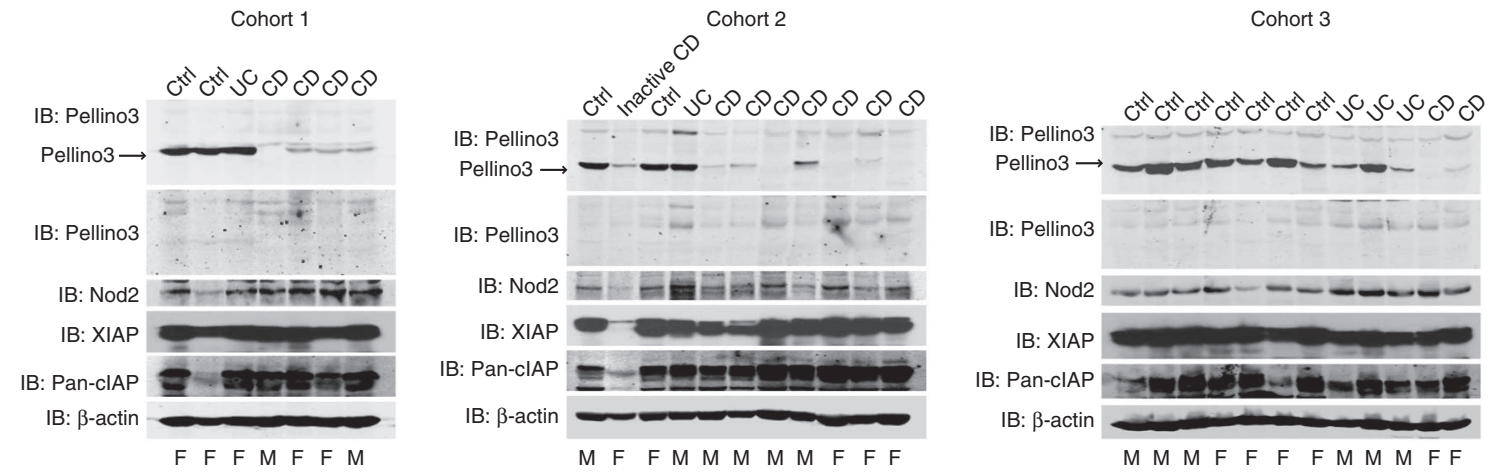

Figure 8 Pellino3 expression is much lower in colons from patients with Crohn's disease. Immunoblot analysis of Pellino3, Nod2, XIAP and clAP1cIAP2 (Pan-clAP) in samples from three independent cohorts (1-3) of male (M) and female (F) healthy control subjects (Ctrl; $n=11)$ and patients with established ulcerative colitis (UC; $n=5$ ) or Crohn's disease (CD; $n=14$ ); second blot in each group, immunoblot analysis with antibody to Pellino3 in the presence of the immunogenic peptide used to generate the anti-Pellino3 (to show immunoreactive bands due to nonspecific binding of antiPellino3). Data are representative of three experiments (one per cohort).

inflammatory bowel disease (IBD), such as Crohn's disease and ulcerative colitis. We searched a publically available database of genome-wide association studies of IBD with the Broad Institute's Ricopili tool for visualizing regions of interest but found no association of single-nucleotide polymorphisms of PELI3 with Crohn's disease or ulcerative colitis. We then used immunoblot analysis to assess the expression of Pellino3 in colonic biopsy samples from healthy controls and patients with Crohn's disease or ulcerative colitis. We consistently found much lower expression of Pellino3 in samples from patients with Crohn's disease than in those from control subjects or patients with ulcerative colitis (Fig. 8). There was no correlation between the expression of Nod2 or IAP in those samples and disease phenotype (Fig. 8). These data suggested that dysregulation of Pellino3 expression may be important in the pathogenesis of Crohn's disease.

\section{DISCUSSION}

Our study has identified Pellino3 as an important mediator of the Nod2 pathway and has extended the understanding of the physiological role of Pellino proteins in the innate immune system. Ubiquitination of RIP2 via K63-linked polyubiquitin chains is a critical step in the Nod2 pathway. Many E2 enzymes (such as the heterodimeric complex Ubc13-Uev1 $a^{15}$ ) and E3 ubiquitin enzymes (cIAP1, cIAP2, xIAP, TRAF6 and Itch ${ }^{15,23-25,45}$ ) have been proposed to catalyze that critical ubiquitination step. Whereas cIAP1 can directly catalyze the attachment of various types of ubiquitin chains to RIP2 with the help of the E2 enzyme UbcH5a ${ }^{45}$, and knockdown of cIAP1 expression results in less ubiquitination of overexpressed RIP2 (ref. 24), pharmacological depletion of cIAP1 does not affect the MDP-induced ubiquitination of RIP2 in wild-type cells ${ }^{23}$. Here we also found that pharmacological depletion of cIAP1 and cIAP2 did not regulate the MDP-induced ubiquitination of RIP2 or downstream activation of NF-KB or MAPK pathways in wild-type BMDMs and that depletion of cIAP had no additional affect on Peli3 ${ }^{-1-}$ BMDMs. A published study has reported that XIAP serves a key role in Nod2 signaling by promoting the ubiquitination of RIP2 and recruitment of the LUBAC to Nod2 (ref. 23). We found that depletion of XIAP augmented the inhibitory effects of Pellino3 deficiency on the Nod2mediated activation of NF- $\mathrm{KB}$ and $\mathrm{p} 38$, which suggested that Pellino3 and XIAP may act in parallel to regulate Nod2 signaling. We propose a model in which Nod2-triggered K63-linked polyubiquitination of RIP2 is critically mediated by Pellino3 and leads to recruitment of the TAK1 and IKK complexes and ensuing downstream signaling. That is closely followed by XIAP-mediated ubiquitination of RIP2, in a nonK63-linked manner, which leads to recruitment of the LUBAC and linear ubiquitination of signaling components that also feed positively into Nod2 downstream signaling. Such a model shares similarity with the TNF signaling pathway, in which early K63-linked ubiquitination of signaling molecules proximal to the TNF receptor is followed by LUBAC-catalyzed linear ubiquitination of components of the receptor complex, which results in stabilization of the signaling complexes and enhanced downstream signaling ${ }^{46}$.

Our study adds to an emerging paradigm in which Pellino proteins target important signaling intermediates in immunological signaling pathways. Our identification of RIP2 as an important target for Pellino3, coupled with a published report describing Pellino1 as the E3 ubiquitin ligase for RIP1 in the TLR3 and TLR4 pathways ${ }^{36}$, emphasizes notable molecular and functional relationships between the Pellino and RIP kinase families. Furthermore, given that Pellino proteins were initially characterized as interaction partners and E3 ubiquitin ligases for IRAKs ${ }^{26}$ and that the latter have roles equivalent to those of RIP kinases in regulating downstream signaling pathways,our study adds to the growing appreciation of the importance of the Pellino family in regulating, by ubiquitination, the function of kinases critically important to innate immunity.

Pellino3 deficiency led to exacerbation of pathology in three independent models of experimental colitis demonstrated before to be mediated by Nod2. Like Nod2-deficient mice ${ }^{40}, P_{\text {eli }}{ }^{-/-}$mice had impaired clearance of $C$. rodentium associated with diminished early inflammatory and $\mathrm{T}_{\mathrm{H}} 1$ and $\mathrm{T}_{\mathrm{H}} 17$ responses and impairment in epithelial and mucosal recovery and greater inflammation at the later stages of colitis. Such enhanced inflammation may mirror the inflammatory phenotype associated with loss-of-function mutations in Nod2 and Crohn's disease. We have also identified a role for Pellino3 in Nod1 signaling, and given that like Nod2, Nod1 has an important role in the response to infection with C. rodentium ${ }^{47}$, some of the in vivo effects of Pellino3 in this model may also be attributed to its regulation of the Nod1 pathway.

We also found substantial downregulation of Pellino3 expression in colon samples from patients with Crohn's disease but not those from patients with ulcerative colitis, which possibly reflects the more selective role of the Nod2 pathway in the former pathology. Whereas mutations and polymorphisms in NOD2 represent the most frequent genetic associations with Crohn's disease, most patients who develop Crohn's disease have the wild-type form of this gene. In those cases, the genetic defects may lie in genes that encode signaling components 
downstream of Nod2. Initial studies of existing data from genomewide association studies did not identify any mutations or polymorphisms in the gene encoding Pellino3, but this does not exclude the possibility that rare variants of PELI3 that are not captured by such studies may be a contributing factor. This should encourage future studies to expand the increasing appreciation of the importance of Pellino proteins in health and disease.

\section{METHODS}

Methods and any associated references are available in the online version of the paper.

Note: Any Supplementary Information and Source Data files are available in the online version of the paper.

\section{ACKNOWLEDGMENTS}

We thank P.A. Barker and M. Saleh (McGill University) for Flag-tagged RIP2 and Nod2; M. Parkers and the Broad Institute for help and use of the Ricopili search tool; and G. Holleran for assistance in collecting biopsy samples from human subjects. Supported by Science Foundation Ireland (07/IN.1/B972 to P.N.M.; 10/IN.1/B3004 to P.G.F.; 02/CE/B124 and 07/CE/B1368 to F.S.; and funding for the Alimentary Pharmabiotic Centre as a Centre for Science, Engineering and Technology) and the Health Research Board of Ireland (PhD/2007/09).

\section{AUTHOR CONTRIBUTIONS}

S.Y. developed the concept, designed and did experiments, analyzed data and prepared the figures; B.W. designed and did experiments and analyzed data; F.H. did the studies involving RIP2 mutants. R.J. and R.B. did the studies of colon explants and primary intestinal epithelial cells. M.E.H. and R.B. did the flow cytometry of cells from mesenteric lymph nodes; G.A. did the DSS and TNBS colitis studies and analyzed data. B.H. and D.M. obtained colon biopsy samples from adult humans and did clinical phenotyping of patients; T.D. and A.Q. did the C. rodentium studies; F.S. and S.M. designed, analyzed and supervised the C. rodentium studies; P.G.F. designed and supervised the DSS and TNBS colitis studies; and P.N.M. conceived of the study, supervised the overall project, analyzed data and wrote the manuscript.

\section{COMPETING FINANCIAL INTERESTS}

The authors declare no competing financial interests.

Reprints and permissions information is available online at http://www.nature.com/ reprints/index.html.

1. Newton, K. \& Dixit, V.M. Signaling in innate immunity and inflammation. Cold Spring Harb. Perspect. Biol. 4, 1-19 (2012).

2. Kawai, T. \& Akira, S. Toll-like receptors and their crosstalk with other innate receptors in infection and immunity. Immunity 34, 637-650 (2011).

3. Rubino, S.J., Selvanantham, T., Girardin, S.E. \& Philpott, D.J. Nod-like receptors in the control of intestinal inflammation. Curr. Opin. Immunol. 24, 398-404 (2012).

4. Hugot, J.P. et al. Association of NOD2 leucine-rich repeat variants with susceptibility to Crohn's disease. Nature 411, 599-603 (2001).

5. Ogura, Y. et al. A frameshift mutation in NOD2 associated with susceptibility to Crohn's disease. Nature 411, 603-606 (2001).

6. Hampe, J. et al. Association between insertion mutation in NOD2 gene and Crohn's disease in German and British populations. Lancet 357, 1925-1928 (2001).

7. Miceli-Richard, C. et al. CARD15 mutations in Blau syndrome. Nat. Genet. 29, 19-20 (2001).

8. Kanazawa, N. et al. Early-onset sarcoidosis and CARD15 mutations with constitutive nuclear factor-kappaB activation: common genetic etiology with Blau syndrome. Blood 105, 1195-1197 (2005).

9. Inohara, N. et al. Host recognition of bacterial muramyl dipeptide mediated through NOD2. Implications for Crohn's disease. J. Biol. Chem. 278, 5509-5512 (2003).

10. Girardin, S.E. et al. Nod2 is a general sensor of peptidoglycan through muramyl dipeptide (MDP) detection. J. Biol. Chem. 278, 8869-8872 (2003).

11. Tanabe, T. et al. Regulatory regions and critical residues of NOD2 involved in muramyl dipeptide recognition. EMBO J. 23, 1587-1597 (2004).

12. Abbott, D.W., Wilkins, A., Asara, J.M. \& Cantley, L.C. The Crohn's disease protein, NOD2, requires RIP2 in order to induce ubiquitinylation of a novel site on NEMO. Curr. Biol. 14, 2217-2227 (2004).

13. Ogura, Y. et al. Nod2, a Nod1/Apaf-1 family member that is restricted to monocytes and activates NF-kB. J. Biol. Chem. 276, 4812-4818 (2001).

14. Abbott, D.W. et al. Coordinated regulation of Toll-like receptor and NOD2 signaling by K63-linked polyubiquitin chains. Mol. Cell Biol. 27, 6012-6025 (2007).
15. Yang, Y. et al. NOD2 pathway activation by MDP or Mycobacterium tuberculosis infection involves the stable polyubiquitination of Rip2. J. Biol. Chem. 282 36223-36229 (2007)

16. Hasegawa, M. et al. A critical role of RICK/RIP2 polyubiquitination in Nod-induced NF- $\kappa B$ activation. EMBO J. 27, 373-383 (2008).

17. McCarthy, J.V., Ni, J. \& Dixit, V.M. RIP2 is a novel NF-KB-activating and cell deathinducing kinase. J. Biol. Chem. 273, 16968-16975 (1998).

18. Windheim, M., Lang, C., Peggie, M., Plater, L.A. \& Cohen, P. Molecular mechanisms involved in the regulation of cytokine production by muramyl dipeptide. Biochem. J. 404, 179-190 (2007).

19. Kanayama, A. et al. TAB2 and TAB3 activate the NF-KB pathway through binding to polyubiquitin chains. Mol. Cell 15, 535-548 (2004).

20. Moynagh, P.N. TLR signalling and activation of IRFs: revisiting old friends from the NF-kappaB pathway. Trends Immunol. 26, 469-476 (2005).

21. Traenckner, E.B. et al. Phosphorylation of human $1 \kappa B-\alpha$ on serines 32 and 36 controls $1 \kappa \mathrm{B}-\alpha$ proteolysis and $\mathrm{NF}-\kappa \mathrm{B}$ activation in response to diverse stimuli. EMBO J. 14, 2876-2883 (1995).

22. Moynagh, P.N. The NF-kB pathway. J. Cell Sci. 118, 4589-4592 (2005).

23. Damgaard, R.B. et al. The ubiquitin ligase XIAP recruits LUBAC for NOD2 signaling in inflammation and innate immunity. Mol. Cell 46, 746-758 (2012).

24. Bertrand, M.J. et al. Cellular inhibitors of apoptosis CIAP1 and CIAP2 are required for innate immunity signaling by the pattern recognition receptors NOD1 and NOD2. Immunity 30, 789-801 (2009).

25. Tao, M. et al. ITCH K63-ubiquitinates the NOD2 binding protein, RIP2, to influence inflammatory signaling pathways. Curr. Biol. 19, 1255-1263 (2009).

26. Moynagh, P.N. The Pellino family: IRAK E3 ligases with emerging roles in innate immune signalling. Trends Immunol. 30, 33-42 (2009).

27. Schauvliege, R., Janssens, S. \& Beyaert, R. Pellino proteins: novel players in TLR and IL-1R signalling. J. Cell Mol. Med. 11, 453-461 (2007).

28. Lin, C.C., Huoh, Y.S., Schmitz, K.R., Jensen, L.E. \& Ferguson, K.M. Pellino proteins contain a cryptic FHA domain that mediates interaction with phosphorylated IRAK1. Structure 16, 1806-1816 (2008).

29. Schauvliege, R., Janssens, S. \& Beyaert, R. Pellino proteins are more than scaffold proteins in TLR/IL-1R signalling: a role as novel RING E3-ubiquitin-ligases. FEBS Lett. 580, 4697-4702 (2006)

30. Butler, M.P., Hanly, J.A. \& Moynagh, P.N. Kinase-active interleukin-1 receptorassociated kinases promote polyubiquitination and degradation of the Pellino family: direct evidence for PELLINO proteins being ubiquitin-protein isopeptide ligases. J. Biol. Chem. 282, 29729-29737 (2007).

31. Ordureau, A. et al. The IRAK-catalysed activation of the E3 ligase function of Pellino isoforms induces the Lys63-linked polyubiquitination of IRAK1. Biochem. J. 409, 43-52 (2008).

32. Smith, H. et al. Identification of the phosphorylation sites on the E3 ubiquitin ligase Pellino that are critical for activation by IRAK1 and IRAK4. Proc. Natl. Acad. Sci. USA 106, 4584-4590 (2009).

33. Smith, $H$. et al. The role of TBKI and IKKE in the expression and activation of Pellino 1. Biochem. J. 434, 537-548 (2011).

34. Goh, E.T. et al. Identification of the protein kinases that activate the E3 ubiquitin ligase Pellino 1 in the innate immune system. Biochem. J. 441, 339-346 (2012).

35. Strelow, A., Kollewe, C. \& Wesche, H. Characterization of Pellino2, a substrate of IRAK1 and IRAK4. FEBS Lett. 547, 157-161 (2003).

36. Chang, M., Jin, W. \& Sun, S.C. Peli1 facilitates TRIF-dependent Toll-like receptor signaling and proinflammatory cytokine production. Nat. Immunol. 10, 1089-1095 (2009).

37. Chang, $M$. et al. The ubiquitin ligase Peli1 negatively regulates $T$ cell activation and prevents autoimmunity. Nat. Immunol. 12, 1002-1009 (2011).

38. Moynagh, P.N. Peli1 (rel)ieves autoimmunity. Nat. Immunol. 12, 927-929 (2011).

39. Siednienko, J. et al. Pellino3 targets the IRF7 pathway and facilitates autoregulation of TLR3- and viral-induced expression of type I interferons. Nat. Immunol. 13 1055-1062 (2012).

40. Kim, Y.G. et al. The Nod2 sensor promotes intestinal pathogen eradication via the chemokine CCL2-dependent recruitment of inflammatory monocytes. Immunity $\mathbf{3 4}$ 769-780 (2011).

41. Murphy, C.T. et al. The sphingosine-1-phosphate analogue FTY720 impairs mucosal immunity and clearance of the enteric pathogen Citrobacter rodentium. Infect. Immun. 80, 2712-2723 (2012).

42. Watanabe, T. et al. Muramyl dipeptide activation of nucleotide-binding oligomerization domain 2 protects mice from experimental colitis. J. Clin. Invest. 118, 545-559 (2008).

43. Hitotsumatsu, 0 . et al. The ubiquitin-editing enzyme A20 restricts nucleotidebinding oligomerization domain containing 2-triggered signals. Immunity $\mathbf{2 8}$ 381-390 (2008).

44. Inohara, N., del Peso, L., Koseki, T., Chen, S. \& Nunez, G. RICK, a novel protein kinase containing a caspase recruitment domain, interacts with CLARP and regulates CD95-mediated apoptosis. J. Biol. Chem. 273, 12296-12300 (1998).

45. Bertrand, M.J. et al. clAP1/2 are direct E3 ligases conjugating diverse types of ubiquitin chains to receptor interacting proteins kinases 1 to 4 (RIP1-4). PLoS ONE 6, e22356 (2011).

46. Haas, T.L. et al. Recruitment of the linear ubiquitin chain assembly complex stabilizes the TNF-R1 signaling complex and is required for TNF-mediated gene induction. Mol. Cell 36, 831-844 (2009).

47. Geddes, K. et al. Identification of an innate T helper type 17 response to intestinal bacterial pathogens. Nat. Med. 17, 837-844 (2011). 


\section{ONLINE METHODS}

Cell culture. HEK293T and HT29 cells were cultured in Dulbecco's modified Eagle's medium. HCT116 cells were cultured in McCoy's 5A medium plus GlutaMAX-I medium (Sigma). THP-1 cells were cultured in RPMI-1640 medium plus GlutaMAX-I medium (Gibco). All growth medium was supplemented with $10 \%$ (vol/vol) FBS (Hyclone), penicillin $(100 \mathrm{U} / \mathrm{ml})$ and streptomycin $(100 \mu \mathrm{g} / \mathrm{ml})$. For isolation of BMDMs, tibias and femurs were removed from wild-type and Peli3 ${ }^{-1-}$ mice by sterile techniques and the bone marrow was flushed with fresh RPMI medium. Cells were plated in medium supplemented with $10 \%$ ( $\mathrm{vol} / \mathrm{vol}$ ) conditioned medium of L929 mouse fibroblasts and were maintained for $4-6 \mathrm{~d}$ at $37^{\circ} \mathrm{C}$ in a humidified atmosphere of $5 \% \mathrm{CO}_{2}$. For isolation of colonic epithelial cells, colons were excised from wild-type and Peli3 ${ }^{-I-}$ mice, washed briefly in PBS and then thoroughly flushed with Hank's balanced-salt solution containing $1 \%(\mathrm{wt} / \mathrm{vol})$ penicillin, $1 \%(\mathrm{wt} / \mathrm{vol})$ streptomycin and gentamicin $(25 \mu \mathrm{g} / \mathrm{ml})$. Colons were opened longitudinally, cut into small segments and washed extensively with Hank's balanced-salt solution before being chopped into a homogenized paste. That paste was incubated at $37^{\circ} \mathrm{C}$ for $1 \mathrm{~h}$ in DMEM containing collagenase $(150 \mathrm{U} / \mathrm{ml})$, hyaluronidase $(150 \mu \mathrm{g} / \mathrm{ml})$ and $1 \%(\mathrm{vol} / \mathrm{vol}) \mathrm{FBS}$ and then was centrifuged at $1,000 \mathrm{~g}$ for 5 min. Pellets were washed with DMEM containing 2\% (wt/vol) sorbitol, and the supernatant containing the purified crypt fraction was collected and centrifuged at $400 \mathrm{~g}$ for $5 \mathrm{~min}$. Crypts were then resuspended in high-glucose DMEM containing $5 \%(\mathrm{vol} / \mathrm{vol}) \mathrm{FBS}, 7.5 \%$ (wt/vol) sodium pyruvate and insulin $(0.25 \mathrm{U} / \mathrm{ml})$ and were plated on collagen-coated plates. The medium was changed every $2 \mathrm{~d}$ and cells were allowed to grow to confluency before being treated. For the preparation of MEFs, $\mathrm{Peli3}^{+/-}$mice were bred to generate wildtype and Peli3 ${ }^{-1-}$ embryos. Embryos were dissected from pregnant females at day 13 after mating. MEFs isolated by standard procedures were cultured in DMEM supplemented with $10 \%$ (vol/vol) FBS and containing penicillin $(100 \mathrm{U} / \mathrm{ml})$ and streptomycin $(100 \mu \mathrm{g} / \mathrm{ml})$. All cell types were maintained in humidified atmosphere a $37^{\circ} \mathrm{C}$ with $5 \% \mathrm{CO}_{2}$.

Colon explants. Colons were excised from wild-type and $\mathrm{Peli3}^{-/-}$mice, washed briefly in PBS and then thoroughly flushed with PBS containing 1\% (wt/vol) penicillin-streptomycin. Colons were opened longitudinally, cut into segments $0.5 \mathrm{~cm}$ in length and washed extensively with PBS containing $1 \%$ (wt/vol) penicillin/streptomycin. Size- and weight-matched explant segments were placed in flat-bottomed 24-well plates in RPMI growth medium and treated.

Expression vectors. Plasmids encoding wild-type Pellino3s or Pellino3l tagged at the carboxy terminus with Myc (pcDNA3.1-Pellino3s-myc or pcDNA3.1Pellino 31-myc) and similarly Myc-tagged mutants with a nonfunctional FHA domain (Pellino3s with substitution of alanine for arginine at position 131 and for serine at position 161 (R131A-S161A) and Pellino3l with substitution of alanine for arginine at position 155 and for serine at position 185 (R155AS185A)) or an altered RING domain (Pellino3s with substitution of alanine for cysteine at positions 360 and 363 (C360A-C363A) and Pellino3l with substitution of alanine for cysteine at positions 384 and 387 C384A/C387A)) and A20 tagged with Flag at the carboxy terminus (A20-Flag) were generated in-house. Hemagglutinin-tagged wild-type ubiquitin and ubiquitin lacking all seven lysine residues or all lysine residues except K6, K11, K27, K29, K33, K48 or K63 were from Addgene. Flag-tagged RIP2 and Nod2 were from P.A. Barker and M. Saleh. Myc-tagged wild-type and mutant (as described above) Pellino3s and Pellino3l constructs were also subcloned into histidine-tagged pRSET-A (Invitrogen). Mouse Pellino3 from mouse embryo brain was cloned into the pcDNA3.1 vector. Mutants of mouse Pellino with a nonfunctional FHA domain (with substitution of alanine for serine at position 161 (S161A)) or an altered RING domain (with substitution of alanine for cysteine at positions 360 and 363 (C360A-C363A) were generated in-house. Glutathione S-transferase-tagged RIP2 (H00008767-P01) was from Novus.

Antibodies and reagents. Anti-IKB $\alpha$ (C-21; sc-371), anti-Ub (P4D1; sc-8017), anti-Tak1 (M-579; sc-7162), anti-IKK $\gamma$ (FL-419; sc-8330) and anti-RIP2 (H-300;sc-22763) were from Santa Cruz; anti-Myc (2276), antibody to phosphorylated IאB $\alpha$ (9246), anti-Jnk(9252), anti-p38 (9212), anti-Erk (9102), antibody to phosphorylated Jnk (9251), antibody to phosphorylated Erk (9101) and antibody to phosphorylated p38 (9211) were from Cell Signaling; anti-HA (16B12;
MMS-101P) was from Covance; antibody to K63-linked ubiquitin (HWA4C4; BML-PW0600) was from Enzo Life Science; antibody to K48-linked ubiquitin (Apu2; 05-1307) was from Millipore; antibody to all mouse cIAP (MAB3400) and anti-XIAP (MAB822) were from R\&D Systems; anti-Flag (F3165) and anti- $\beta$-actin (AC-15;A 1978) were from Sigma; polyclonal antibody to Sharpin (14626-1-AP) was from Proteintech; rabbit anti-Pellino3 was generated inhouse; IRDye 680RD-anti-mouse (926-68070) and IRDye 800CW-anti-rabbit (926-32211) were from LI-COR Biosciences; IRDye 800CW-anti-goat (605-731-002) was from Rockland; horseradish peroxidase-anti-mouse (115035-008) and horseradish peroxidase-anti-rabbit (211-032-171) were from Jackson ImmunoResearch; and fluorescein isothiocyanate-anti-CD4 (GK1.5), phycoerythrin-anti-CD11b (M1/70; 12-0112), allophycocyanin-anti-Gr-1 (RB6-8C5; 17-5931-81) and allophycocyanin-anti-IFN- $\gamma$ (XMG1.2; 17-7311) and the isotype-matched control antibodies phycoerythrin-conjugated rat IgG2b $\kappa$-chain (Eb149/10H5; 12-4031), allophycocyanin-conjugated rat IgG2b $\kappa$-chain (Eb149/10H5; 17-4031), allophycocyanin-conjugated rat IgG1 $\kappa$-chain (eBRG1; 17-4301), phycoerythrin-conjugated rat IgG1 $\mathrm{\kappa}$-chain (eBRG1; 12-4301) and fluorescein isothiocyanate-conjugated rat IgG2a $\kappa$-chain (eBR2a; 11-4321) were from eBioscience. Fluorescein isothiocyanate-anti-F4/80 (BM8; 123107) was from Biolegend. Allophycocyanin-anti-CD3 (145-2C11; 553066), phycoerythrin-anti-IL-17 (TC11-18H10; 559502) and allophycocyaninconjugated Armenian hamster IgG1 $\kappa$-chain (isotype-matched control antibody; A19-3; 553974) were from Becton Dickenson. EasyBlocker and EasyBlot horseradish peroxidase-conjugated antibody to rabbit IgG (GTX221666-01) were from GeneTex; MDP (Ac-muramyl-Ala-D-Glu-NH $\mathrm{N}_{2}$; G-1055) was from Bachem. MDP was transfected into cells through the use of Lipofectamine 2000. Pellino3-specific siRNA (sense, 5'-GCACAGCAUCUCGUAUAC ATT-3'; antisense, 5'-UGUAUACGAGAUGCUGUGCTG3-') was from Ambion and cells were transfected through the use of Lipfectamine 2000 according to the manufacturer's instructions (Invitrogen). LPS was from Enzo Life Sciences. $\mathrm{Pam}_{3} \mathrm{CSK}_{4}$ (tripalmitoyl cysteinyl seryl tetralysine lipopeptide) was from Invivogen.

Knockdown of Pellino3 by lentiviral shRNA in human cells. Lentiviral constructs encoding human Pellino3-specific shRNA specifically targeted the 3 ' untranslated region of PELI3 and had the following sequence: GCACTTG CTGATAGCCACTATCCGGGCACTTGCTGATAGCCACTATCTCGAGAT AGTGGCTATCAGCAAGTGCTTTTTTG. Constructs were transfected into HEK293T cells together with packaging and envelope plasmids (dR89.1 and VSV-G), and lentivirus from supernatants of the medium was used to infect THP- 1 cells. Cells were then grown for $4 \mathrm{~d}$ under puromycin $(10 \mu \mathrm{g} / \mathrm{ml})$ selection. Knockdown efficiency was assessed by immunoblot analysis of Pellino3 in whole-cell lysates. Control cells were infected with lentivirus containing a nontargeting vector that encodes shRNA that does not match the sequence of any known human or mouse gene. For rescue assays, Plv lentiviral vectors encoding Myc-tagged wild-type or mutant Pellino3l were transfected into HEK293T cells along with packaging and envelope plasmids (pPTK and pMDG). Plv lentivirus from the medium supernatants was used for infection THP-1 cells along with shRNA lentivirus. Cells were grown for $4 \mathrm{~d}$ under puromycin $(10 \mu \mathrm{g} / \mathrm{ml})$ selection before experiments.

Mice. Peli $3^{-/-}$mice were generated on a C57BL/6J background as described ${ }^{39}$ Nod2 $2^{-/-}$mice were generated on a C57BL/6J strain background, and wildtype C57BL/6J mice originally from Jackson Laboratories were bred in-house. All animal experiments were done in accordance with the regulations and guidelines of the Irish Department of Health and protocols were approved by the Research Ethics committees of National University of Ireland Maynooth, Trinity College Dublin and the University College Cork Animal Experimentation Ethics Committee.

Chemically induced colitis models. Two mouse models of chemically induced intestinal inflammation were generated by the administration of DSS or TNBS. Colitis was induced by DSS as described ${ }^{48}$. DSS (35,000-50,000 kDa; MP Biomedicals) was dissolved in the drinking water provided to mice. Fresh DSS solution was provided every second day. Wild-type, Peli3 ${ }^{-/-}$and $\mathrm{Nod}^{-1-}$ mice received $2.5 \%$ DSS for $5 \mathrm{~d}$, followed by 'normal' drinking water (without DSS) for $3 \mathrm{~d}$. On days 0,1 , and 2, mice were also given intraperitoneal injection 


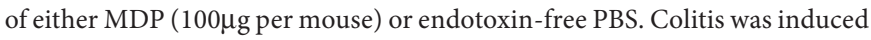
by TNBS as described ${ }^{49}$. TNBS solution ( $100 \mu \mathrm{l}$ volume; $3.75 \mathrm{mg}$ per mouse in $45 \%$ ( $\mathrm{vol} / \mathrm{vol})$ ethanol) or vehicle control solution ( $45 \%$ ethanol) was administered into the colon lumen of anesthetized mice by insertion of a catheter $4 \mathrm{~cm}$ proximal to the anus. After administration of DSS or TNBS, mice were checked each day for morbidity and body weight. At autopsy, the lengths of the colons were measured and a segment of distal colon $\sim 1 \mathrm{~cm}$ in length was removed and fixed in $10 \%$ formaldehyde in saline. Tissue was embedded in paraffin and sections $5 \mu \mathrm{m}$ in thickness were stained with hematoxylin and eosin. Histology scores were assigned by researchers 'blinded' to sample identity, according to the following scoring system: a maximum combined score of 10 was obtained from the amount of inflammatory cell infiltration ( 0 , none; 1 , slightly dispersed cell infiltrate; 2 , moderately increased cell infiltrates forming occasional cell foci; and 3 , severely large areas of cell infiltrates causing loss of tissue architecture), extent of injury ( 0 , none; 1 , mucosal; 2 , mucosal and submucosal; and 3 , transmural), and crypt damage ( 0 , none; 1 , basal one-third damaged; 2, basal two-thirds damaged; 3 , only surface epithelium intact; and 4 , loss of entire crypt and epithelium).

C. rodentium infection and bioluminescent imaging of mice. Mice were randomly separated into groups of eight to nine mice and were given $1 \times 10^{10}$ CFU of the bioluminescent $C$. rodentium derivative strain ICC 180 by oral gavage and then were monitored for changes in body weight and clinical signs of disease as described ${ }^{41,50}$. Bacteria were counted in fecal samples collected at various time points and in homogenates of spleen, liver and mesenteric lymph nodes collected when mice were killed on days 12 and 21 after infection as described ${ }^{41,50}$. In vivo bioluminescence imaging of whole body and colons was done with an IVIS 100 charge-coupled-device imaging system (Xenogen). Bioluminescent signals were quantified for regions of interest over the same surface area of the whole body and colons. Background was omitted by the measurement of signal intensity in regions of interest of whole body and colons of uninfected mice and subtraction of that from the results obtained for infected mice. Imaging data were analyzed and quantified with Living Image software (version 2.50; Xenogen) and are presented as the number of photons per second per $\mathrm{cm}^{2}$.

After colons were imaged, the length and weight of colon was measured. The distal $3 \mathrm{~cm}$ were divided longitudinally. One piece was frozen in liquid nitrogen and later thawed, homogenized and analyzed with a Mouse ProInflammatory 7-Plex Ultra-Sensitive kit (Meso Scale Discovery) to assess IFN- $\gamma$, TNF, IL-1 $\beta$, IL-6, IL-10, IL-12p70 and keratinocyte chemoattractant and by ELISA (R\&D Systems) to assess CCL2, each according to the manufacturer's instructions. The other piece of distal colon was rolled (like a 'Swiss roll') and fixed in 10\% formalin, followed by embedding in paraffin as described above.

Staining with hematoxylin and eosin and periodic acid Schiff stain. Sections of distal colon $5 \mu \mathrm{m}$ in thickness were stained with hematoxylin and eosin or with periodic acid Schiff stain according to standard histological procedures. Each whole-colon 'Swiss roll' was evaluated by a researcher (S.M.) 'blinded' to sample identity, and scores were assigned on a scale of 0 to 3 ( 0 , none; 1 , mild; 2 , moderate; 3 , severe) for inflammatory damage, such as goblet cell loss, crypt elongation, mucosal thickening, and epithelial injury, including hyperplasia and shedding of enterocytes into the gut lumen; this yielded a maximum score of 12 per mouse section ${ }^{41}$. For mucin staining, a score based on the number and size of mucin-containing cells, on a scale of 0 to 3, was assigned as described above; this yielded a maximum score of 6 per section.

Flow cytometry. Mesenteric lymph nodes were dissected, homogenized in RPMI medium and centrifuged for $5 \mathrm{~min}$ at $1500 \mathrm{~g}$ and $4{ }^{\circ} \mathrm{C}$. Supernatants were discarded and red blood cells were lysed in $0.15 \mathrm{M} \mathrm{NH}_{4} \mathrm{Cl}, 1 \mathrm{mM} \mathrm{KHCO}_{3}$ and 0.1 mM EDTA, pH 7.2, followed by dilution in RPMI medium and further centrifugation as described above. Cell pellets were resuspended in RPMI medium $\left(1 \times 10^{6}\right.$ cells per $\left.\mathrm{ml}\right)$. Aliquots of cell suspensions $(200 \mu \mathrm{l}$ in volume $)$ were transferred to V-bottomed 96 -well plates and stained with phycoerythrin-antiCD11b (M1/70; 12-0112; eBioscience), allophycocyanin-anti-Gr-1 (RB6-8C5; 17-5931-81; eBioscience), fluorescein isothiocyanate-anti-F4/80 (BM8; 123107; Biolegend), allophycocyanin-anti-CD3 (145-2C11; 553066; BD) or fluorescein isothiocyanate-anti-CD4 (GK1.5; eBioscience), and expression was assessed by flow cytometry with an Accuri C6 (BD). Cells were also fixed and made permeable with a commercial kit (eBioscience) and intracellular cytokines were analyzed with allophycocyanin-anti-IFN- $\gamma$ (XMG1.2; 17-7311; eBioscience) and phycoerythrin-anti-IL-17 (TC11-18H10; 559502; BD). Nonspecific staining was defined through the use of the following isotype-matched control antibodies with the respective fluorochrome label: phycoerythrin-conjugated rat IgG2b K-chain (Eb149/10H5;12-4031), allophycocyanin-conjugated rat IgG2b K-chain (Eb149/10H5; 17-4031), allophycocyanin-conjugated rat IgG1 $\kappa$-chain (eBRG1; 17-4301), phycoerythrin-conjugated rat IgG1 $\kappa$-chain (eBRG1; 12-4301) and fluorescein isothiocyanate-conjugated rat IgG2a $\kappa$-chain (eBR2a; 11-4321; all from eBioscience); and allophycocyanin-conjugated Armenian hamster IgG1 к-chain (A19-3; 553974; BD).

Luciferase reporter assay. HEK293T cells $\left(1.2 \times 10^{5}\right.$ cells per $\left.\mathrm{ml} ; 200 \mu \mathrm{l}\right)$ and MEFs $\left(0.8 \times 10^{5}\right.$ cells per $\left.\mathrm{ml} ; 200 \mu \mathrm{l}\right)$ were seeded in 96 -well plates. Cells were cotransfected, through the use of Lipofectamine 2000 transfection reagent, with plasmids encoding NFKB-regulated firefly luciferase and thymidine kinase-controlled renilla luciferase (Promega Biosciences) and designated amounts of expression constructs encoding Nod2 and various forms of Pellino3. Cells were allowed to grow for another $24 \mathrm{~h}$ and then treated for $18 \mathrm{~h}$ with MDP. Cell extracts were collected in Reporter Lysis Buffer (Promega Biosciences), and firefly and renilla luciferase activity was assayed with the Luciferase Assay system (Promega Biosciences) and coelenterazine $(1 \mu \mathrm{g} / \mathrm{ml}$, Insight Biotechnology), respectively. In some experiments, cells were initially transfected with siRNA $24 \mathrm{~h}$ before further transfection with the expression constructs described above.

Real-time PCR assays. Primary BMDMs $\left(1.5 \times 10^{6}\right.$ cells per $\left.\mathrm{ml} ; 2 \mathrm{ml}\right)$ and MEFs $\left(1.2 \times 10^{5}\right.$ cells per $\left.\mathrm{ml} ; 2 \mathrm{ml}\right)$ were seeded into 12 -well plates. After the appropriate treatment(s), RNA was extracted with TRIzol (Invitrogen). Then, cDNA was generated from RNA $(2 \mu \mathrm{g})$ with MMLV Reverse Transcriptase (Bioscript Bioline) and real-time PCR analyses were done with Brilliant SYBR Green QPCR Master mix according to the manufacturer's instructions (Stratagene). The following primers were used: mouse Il6, forward, ACAACCACGGCCTTCCCTAC, and reverse, TCCACGATTTCCCAGAGAACA; mouse $I l 1 b$, forward, TTGAA GTTGACGGACCCCA, and reverse, ATGAGTGATACTGCCTGCCTGA; mouse Tnf, forward, CATCTTCTCAAAATTCGAGTGA, and reverse, TGG GAGTAGACAAGGTAC; mouse $I l 12 b$, forward, TGGTTTGCCATCGTTTT GC, and reverse, GGGAGTCCAGTCCACCTCTACA; and mouse Cxcl1, forward, CACCCAAACCGAAGTCATAGC, and reverse, TTGGGGACACC TTTTAGCATC. The abundance of each mRNA was normalized relative to PCR of the housekeeping gene Hprt with the following primers: forward, GTCCCAGCGTCGTGATTAGC, and reverse, TGGCCTCCCAT CTCCTTCA.

Cytokine ELISA. Primary BMDMs were seeded $\left(1.5 \times 10^{6}\right.$ cells per $\left.\mathrm{ml} ; 2 \mathrm{ml}\right)$ in 12-well plates and were allowed to grow for $24 \mathrm{~h}$. Cells were then stimulated for $24 \mathrm{~h}$ with MDP $(50 \mu \mathrm{g} / \mathrm{ml})$. Conditioned medium was collected, and IL- $1 \beta$, IL-6, TNF, IL12p40 and CXCL1 were measured by sandwich ELISA (R\&D Systems). Colon explants and primary mouse colonic epithelial cells isolated from wild-type and Peli3-/- mice were left untreated or were stimulated for $16 \mathrm{~h}$ or $24 \mathrm{~h}$ with MDP $(50 \mu \mathrm{g} / \mathrm{ml})$. The medium was collected and IL-6, CXCL1 and CCL2 proteins were analyzed. For MEFs, cells were seeded $\left(1.2 \times 10^{5}\right.$ cells per $\mathrm{ml} ; 2 \mathrm{ml}$ ) in 12-well plates and were allowed to grow for $24 \mathrm{~h}$. At $24 \mathrm{~h}$ after transfection, cells were treated for $18 \mathrm{~h}$ with MDP and then conditioned medium was collected for assay. HCT116, THP-1 and HT29 cells with and without (control) knockdown of Pellino3 were treated for $24 \mathrm{~h}$ with MDP $(50 \mu \mathrm{g} / \mathrm{ml})$ or diaminopimelic acid $(50 \mu \mathrm{g} / \mathrm{ml})$ and human IL-6, IL-8, TNF and CXCL1 (R\&D Systems) were measured in the medium. For analysis of cytokine abundance in mice challenged with MDP, wild-type and $\mathrm{Peli}^{-/-}$mice were given intraperitoneal injection of MDP ( $25 \mathrm{mg}$ per kg body weight) and blood was collected $4 \mathrm{~h}$ after injection. IL-6, keratinocyte chemoattractant, IL-12p 40 , IL-1 $\beta$, IL-10 and TNF were measured in the serum by the Multi-spot assay system, according to the manufacturer's instructions (MSD).

Immunoprecipitation and immunoblot analysis. Primary BMDMs $\left(2 \times 10^{6} \mathrm{cells}\right.$ per $\mathrm{ml} ; 10 \mathrm{ml})$ and MEFs $\left(2 \times 10^{5}\right.$ cells per $\left.\mathrm{ml} ; 10 \mathrm{ml}\right)$ were grown in 
10-cm dishes. Cells lines were grown in six-well plates and in some cases were transfected with the appropriate expression constructs. Cells were then collected in $500 \mu \mathrm{l}$ lysis buffer $(20 \mathrm{mM}$ Tris- $\mathrm{HCl}, \mathrm{pH} 7.4$, containing $150 \mathrm{mM}$ $\mathrm{NaCl}, 1 \%$ (vol/vol) Igepal, 10\% (wt/vol) glycerol, $50 \mathrm{mM} \mathrm{NaF}, 1 \mathrm{mM} \mathrm{Na} 3 \mathrm{VO}$, $1 \mathrm{mM}$ dithiothreitol, $1 \mathrm{mM}$ phenylmethylsulfonyl fluoride and complete protease-inhibitor 'cocktail' (Roche)), followed by incubation for $30 \mathrm{~min}$ at $4{ }^{\circ} \mathrm{C}$. Cell lysates were initially precleared with nonimmune IgG antibody $(1 \mu \mathrm{g}$; mouse IgG control (sc-2025; Santa Cruz) or rabbit IgG control (sc-2027; Santa Cruz)) and protein A-protein G-agarose $(10 \mu \mathrm{l})$, followed by incubation for $24 \mathrm{~h}$ at $4{ }^{\circ} \mathrm{C}$ with the appropriate antibody $(2 \mu \mathrm{g}$ each: anti-Myc (2276; Cell Signaling) or anti-RIP2 (H-300; sc-22763; Santa Cruz)). An aliquot (50 $\mu \mathrm{l})$ of protein A-protein $\mathrm{G}$-agarose was added to each sample, followed by incubation overnight at $4{ }^{\circ} \mathrm{C}$. Immunoprecipitates were collected by centrifugation for $1 \mathrm{~min}$ at $1,000 \mathrm{~g}$ and $4{ }^{\circ} \mathrm{C}$ and the beads were then washed four times with $500 \mu \mathrm{l}$ lysis buffer (without Na3VO4, dithiothreitol, phenylmethylsulfonyl fluoride or protease-inhibitor 'cocktail'). An aliquot $(50 \mu \mathrm{l})$ of SDS-PAGE sample buffer (62.5 mM Tris-HCl, pH 6.8, 10\% (wt/vol) glycerol, 2\% (wt/vol) SDS, $0.7 \mathrm{M} \beta$-mercaptoethanol and $0.001 \%$ (wt/vol) bromophenol blue) was added to the beads. Samples were resolved by SDS-PAGE, transferred to nitrocellulose membranes and analyzed by immunoblot with the appropriate antibodies as follows: anti-RIP2 (H-300; sc-22763; Santa Cruz), anti-Myc (2276; Cell Signaling), anti-ubiquitin (P4D1; sc-8017; Santa Cruz), anti-hemagglutinin (16B12; MMS-101P; Covance), anti-TAK1 (M-579; sc-7162; Santa Cruz), anti-IKK $\gamma$ (FL-419; sc-8330; Santa Cruz), antibody to K63-linked ubiquitin (HWA4C4; BML-PW0600; Enzo Life Science), antibody to K48-linked ubiquitin (Apu2; 05-1307; Millipore), polyclonal antibody to Sharpin (14626-1-AP; Proteintech) and anti-Pellino3 (generated in-house). Immunoreactivity was visualized by the Odyssey Imaging System (LI-COR Biosciences) or enhanced chemiluminescence. For experiments assesing the ubiquitination status of RIP2, cells were collected in $100 \mu$ l lysis buffer $(20 \mathrm{mM}$ Tris- $\mathrm{HCl}, \mathrm{pH} 7.4$, containing $150 \mathrm{mM} \mathrm{NaCl}, 1 \%$ (vol/vol) Igepal, $10 \%$ (wt/vol) glycerol, $50 \mathrm{mM} \mathrm{NaF}$, $1 \mathrm{mM} \mathrm{Na} 3 \mathrm{VO} 4,1 \mathrm{mM}$ dithiothreitol, $1 \mathrm{mM}$ phenylmethylsulfonyl fluoride and complete protease-inhibitor 'cocktail' (Roche)). Cell lysates were treated with $1 \%\left(\mathrm{wt} / \mathrm{vol}\right.$ ) SDS and were heated to $95^{\circ} \mathrm{C}$ for $5 \mathrm{~min}$ to dissociate RIP2 from any associated proteins. Lysates were then diluted tenfold in lysis buffer before immunoprecipitation and immunoblot analysis as described above.

Generation of recombinant proteins and in vitro binding assays. Myc-tagged Pellino3s and Pellino3l (wild-type and mutants as described above) were cloned into the histidine-tagged vector pRSET-A (Invitrogen). The constructs were transformed into Escherichia coli cells, protein expression was induced by isopropyl $\beta$-D-thiogalactopyranoside and recombinant proteins were purified from cell lysates with nickel-nitrilotriacetic acid-agarose according to the manufacturer's instructions (Qiagen). The purity and integrity of recombinant proteins were confirmed by SDS-PAGE followed by Coomassie staining, in addition to immunoblot analysis with anti-Myc (2276; Cell Signaling). For in vitro binding assays characterizing the binding of Pellino3 to partner proteins, recombinant Myc-tagged Pellino3 $(1 \mu \mathrm{g})$ was initially immunoprecipi-

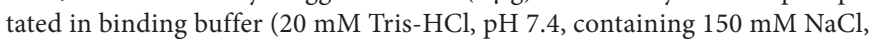
$0.2 \%$ (vol/vol) Igepal, $10 \%$ (wt/vol) glycerol and complete protease-inhibitor 'cocktail') with anti-Myc ( $2 \mu \mathrm{g} ; 2276$; Cell Signaling) and protein A-protein G beads $(30 \mu \mathrm{l})$ or $\mathrm{Ni}^{+}$beads $(15 \mu \mathrm{l})$. The beads were washed five times with binding buffer and then incubated overnight at $4{ }^{\circ} \mathrm{C}$ with lysates $(300 \mu \mathrm{l}$ per well of six-well plates) from HEK293 cells that had been transfected to express Flag-tagged RIP2 or recombinant glutathione $S$-transferase-RIP2 (500 ng). Beads were subjected to serial washes with binding buffer followed by centrifugation. Coprecipitated proteins were eluted in SDS-PAGE sample buffer (62.5 mM Tris-HCl, pH 6.8, 10\% (wt/vol) glycerol, 2\% (wt/vol) SDS, $0.7 \mathrm{M}$ $\beta$-mercaptoethanol and $0.001 \%(\mathrm{wt} / \mathrm{vol}$ ) bromophenol blue) and then analyzed by immunoblot .

In vitro ubiquitination assay. Recombinant Pellino3s (wild-type or mutants as described above; $0.5 \mu \mathrm{g}$ ) was incubated with recombinant RIP2 (400 ng), ubiquitin with substitution of all lysine residues except K63 or with the K63R substitution $(4 \mu \mathrm{g}), \mathrm{E} 1(100 \mathrm{nM}), \mathrm{UbcH} 13-\mathrm{Uevla}(500 \mathrm{nM})$ and a protease inhibitor mixture (EDTA-free) in $20 \mathrm{mM}$ Tris- $\mathrm{HCl}, \mathrm{pH} 8$, containing $2 \mathrm{mM} \mathrm{MgCl} 2,2 \mathrm{mM}$ ATP and $100 \mathrm{mM} \mathrm{NaCl}$. Reactions were incubated at $30^{\circ} \mathrm{C}$ for $2 \mathrm{~h}$ and terminated by the addition of $1 \%$ (wt/vol) SDS. Samples were heated to $95^{\circ} \mathrm{C}$ for $5 \mathrm{~min}$ to dissociate RIP2 from any associated proteins and then were diluted tenfold in lysis buffer $(20 \mathrm{mM}$ Tris- $\mathrm{HCl}$, pH 7.4, containing $150 \mathrm{mM} \mathrm{NaCl}, 1 \%$ (vol/vol) Igepal, $10 \%$ (wt/vol) glycerol, $50 \mathrm{mM} \mathrm{NaF}$, $1 \mathrm{mM} \mathrm{Na}_{3} \mathrm{VO}_{4}, 1 \mathrm{mM}$ dithiothreitol, $1 \mathrm{mM}$ phenylmethylsulfonyl fluoride and complete protease-inhibitor 'cocktail' (Roche)) before immunoprecipitation and immunoblot analysis.

Analysis of human colon samples from patients with Crohn's disease. Human subjects $18-80$ years of age who were undergoing routine colonoscopy as part of their ongoing care were prospectively recruited for this study (approved by The Adelaide and Meath/St James's Hospital Joint Ethics Committee). After informed consent was obtained, two additional biopsies were collected from the abnormal (inflamed) colonic or ileal segment of patients with IBD and from the left colon in control subjects. Biopsies ('anonymous'; without identifying information) were 'snap-frozen' and stored at $-80^{\circ} \mathrm{C}$ for batch analysis. Patient demographics, medical history, endoscopy findings and routine histology were recorded. A total of 30 subjects were enrolled: 19 (63\%) with IBD and 11 (37\%) controls. Of those 30, $14(47 \%)$ were male and the mean age was 46 years \pm 16.4 years. There were more women in the control group ( 7 of $11 ; 63 \%$ ) than in the group with IBD ( 9 of $19 ; 47 \%$; $P<0.05$ ). The controls were slightly older (mean age, 54.16 years \pm 16.2 years; range, $18-73$ years) than were the patients with IBD (mean age, 43.9 years \pm 16.2 years; range, $25-73$ years), but this did not reach statistical significance $(P<0.24)$. Control subjects were defined as subjects with a normal ileo-colonoscopy and negative histology. The control subjects did not have symptoms suggestive of IBD. Of the control subjects, 8 were undergoing colonoscopy for polyp surveillance and the remaining 3 had anemia. In the group with IBD, 14 had established Crohn's disease ( 10 with ileo-colonic disease and 4 with isolated ileal disease (ileal and ileo-colonic diseases are two varieties of Crohn's and represent the site of activity)) and 5 had ulcerative colitis. Among the patients with IBD, $15(79 \%)$ were on maintenance therapy for IBD, including $5(26 \%)$ receiving adalimumab, 7 (37\%) receiving aminosalycilic acid and 3(16\%) receiving oral steroids. By endoscopy, the inflammation was assigned a grade of 'moderate' for $6(32 \%)$ of the patients with IBD and as 'mild' for the remaining 13 (68\%) patients with IBD. Routine histology confirmed active inflammation in 12 cases $(63 \%)$. Histology was normal for all 11 control subjects.

Frozen biopsy samples were thawed and placed in cell lysis buffer $(20 \mathrm{mM}$ Tris- $\mathrm{HCl}$, pH 7.4, containing $150 \mathrm{mM} \mathrm{NaCl}, 1 \%$ (vol/vol) Igepal, 10\% (wt/vol) glycerol, $50 \mathrm{mM} \mathrm{NaF}, 1 \mathrm{mM} \mathrm{Na} 3 \mathrm{VO} 4,1 \mathrm{mM}$ dithiothreitol, $1 \mathrm{mM}$ phenylmethylsulfonyl fluoride and complete protease-inhibitor 'cocktail' (Roche)), followed by sonication. Cell lysates were centrifuged for $10 \mathrm{~min}$ at $14,000 \mathrm{~g}$ for removal of cell debris and nuclei. Supernatants were assayed for protein concentration and lysate samples $(50 \mu \mathrm{g})$ were resolved by SDS-PAGE, transferred to nitrocellulose membranes and analyzed by immunoblot with anti-Pellino3 (generated in-house), anti-Nod2 (sc-56168; Santa Cruz), anti-XIAP (MAB822; R\&D Systems), antibody to all cIAP proteins (MAB3400; R\&D Systems) and anti- $\beta$-actin (loading control; AC-15; Sigma). For anti-Pellino3, immunoblot analysis was done in the absence or presence of the immunogenic peptide that was used to generate the anti-Pellino3, to 'define' the nonspecific binding of the antibody. Immunoreactivity was visualized by the Odyssey Imaging System (LI-COR Biosciences) or enhanced chemiluminescence.

Statistical analysis. Prism5 software (GraphPad Software) was used for all statistical tests. A $P$ value of 0.05 was considered significant.

48. Smith, P. et al. Infection with a helminth parasite prevents experimental colitis via a macrophage-mediated mechanism. J. Immunol. 178, 4557-4566 (2007).

49. Wirtz, S., Neufert, C., Weigmann, B. \& Neurath, M.F. Chemically induced mouse models of intestinal inflammation. Nat. Protoc. 2, 541-546 (2007).

50. Hall, L.J. et al. Natural killer cells protect mice from DSS-induced colitis by regulating neutrophil function via the NKG2A receptor. Mucosal Immunol. advance online publication, doi:10.1038/mi.2012.140 (23 January 2013). 